\title{
Gapless color superconductivity at zero and at finite temperature
}

\author{
Mei Huang ${ }^{1}$ and Igor Shovkovy ${ }^{2, *}$ \\ Institut für Theoretische Physik, J.W. Goethe-Universität, D-60054 \\ Frankurt/Main, Germany
}

\begin{abstract}
We present a detailed study of the recently proposed gapless color superconductivity in neutral two-flavor quark matter in $\beta$-equilibrium at zero as well as at finite temperature. We clarify the nature of the fermionic quasiparticles, and discuss the physical properties of this gapless phase of quark matter. The appearance of two "secondary" spin-1 condensates in the gapless ground state is pointed out. The gluon screening properties are also discussed. It is found that finite temperature properties of the gapless color superconductor are rather unusual. One of the most striking results is that the ratio of the critical temperature to the gap at zero temperature is not a universal number. Moreover, this ratio could become arbitrarily large at some values of the coupling constant. In addition, the value of the gap parameter is not a monotonic function of temperature, although the phase transition is a second order phase transition. In an extreme case, in fact, a nonzero finite temperature gap can appear even if its zero temperature value is vanishing.
\end{abstract}

Key words: Gapless color superconductor, dense quark matter, compact stars, Anderson-Higgs mechanism, Meissner effect

PACS: 12.38.-t, 26.60.+c

e-Print archive number: hep-ph/0307273

* Corresponding author. Full postal address:

Institut für Theoretische Physik,

J.W. Goethe-Universität,

Postfach 1119 32,

D-60054 Frankurt/Main, Germany

Tel.:(+49) (69) 798-23310, Fax: (+49) (69) 798-28350

Email addresses: huang@th.physik.uni-frankfurt.de (Mei Huang), shovkovy@th.physik. uni-frankfurt.de (Igor Shovkovy).

1 On leave from Physics Department, Tsinghua University, Beijing 100084, China.

2 On leave from Bogolyubov Institute for Theoretical Physics, 03143, Kiev, Ukraine. 


\section{Introduction}

Sufficiently cold and dense quark matter is a color superconductor [1]. The corresponding ground state is characterized by a condensate of Cooper pairs made of quarks. Since the latter carry color charge, the $\mathrm{SU}(3)_{c}$ color gauge group of strong interactions is partially or completely broken through the Anderson-Higgs mechanism. At asymptotic densities, this phenomenon was studied from first principles in Refs. $[2,3,4]$. These studies of dense QCD, as well as studies of various phenomenologically motivated models $[5,6,7,8,9]$, suggest that dense quark matter at high baryon density has a very rich phase structure, consisting of many different phases.

It is natural to expect that some color superconducting phases may exist in the interior of compact stars. The estimated central densities of such stars might be as large as $10 \rho_{0}$ (where $\rho_{0} \approx 0.15 \mathrm{fm}^{-3}$ is the saturation density), while their temperatures are in the range of tens of $\mathrm{keV}$. This could provide ideal conditions for the diquark Cooper pairing of color superconductivity.

Matter in the bulk of compact stars should be neutral with respect to electrical as well as color charges. Also, such matter should remain in $\beta$-equilibrium. Satisfying these requirements may impose nontrivial relations between the chemical potentials of different quarks. In turn, such relations could substantially influence the pairing dynamics between quarks, for instance, by suppressing some color superconducting phases and by favoring others. For example, it was argued in Ref. [10], that the mixture of the 2-flavor color superconducting (2SC) phase and normal strange quarks is less favorable than the color-flavorlocked (CFL) phase if the charge neutrality condition is enforced (note that the strange quark mass was chosen too small to allow the appearance of a pure 2SC phase). A similar conclusion was also reached in Ref. [11].

Recently, it was proposed that neutral two-flavor quark matter in $\beta$-equilibrium can have a rather unusual, stable ground state called a gapless color superconductor (g2SC) [12]. A similar phase of gapless color superconductivity could also appear in strange quark matter when the baryon number density is kept fixed [13] [for earlier studies see Ref. [14] where an unstable gapless phase was considered]. This gapless strange quark matter may, in principle, be realized inside the so-called strangelets. It was also suggested that closely related phases may exist in two component mixtures of cold fermionic atoms [15]. It is fair to mention that, in both quark and cold atom systems, the ground state could also be given by some mixed phases $[16,17,18]$ provided the surface tension effects are not too strong.

While the symmetry of the g2SC ground state is the same as that of the conventional 2-flavor color superconductor, the spectrum of the fermionic quasi- 
particles is different. In particular, two out of four gaped quasiparticles of the conventional 2SC phase become gapless in the g2SC phase. In addition, the number densities of the pairing quarks in g2SC at zero temperature are not equal [12]. For example, the density of red (green) up quarks is different from the density of green (red) down quarks. This is in contrast to the conventional "enforced" pairing [19] where the corresponding densities are equal.

It is possible that the g2SC quark matter exists inside central regions of compact stars. This would be quite natural since this phase of matter is neutral with respect to electrical and color charges, and satisfies the $\beta$-equilibrium condition by construction. Also, this phase is more stable than normal quark matter under similar conditions [12]. While suggestive, these arguments alone are not sufficient to make a conclusive statement regarding the chances of the g2SC phase to appear inside stars. In order to study this issue properly, one should consider the competition of the g2SC phase with all other possible phases of quark/hadronic matter. However, the limited knowledge of the strongly coupled dynamics in QCD at realistic densities does not allow such a systematic quantitative study.

In this paper, we study properties of the g2SC phase at finite temperature. Our interest in such properties is driven by the expectation that the g2SC phase of quark matter may already appear at a relatively early (protoneutron) stage of the compact star evolution when the temperature of the stellar core is quite high. If this is the case, the knowledge of finite temperature properties of quark matter would be needed to understand the stellar properties and, possibly, even to predict observational signatures of the new state of matter inside stars.

This paper is organized as follows. In the next section, we set our notation by presenting a model of quark matter, and spell out the main assumptions in the analysis. Then, in Sec. 3 we discuss in detail the solution to the gap equation and the condition of the charge neutrality in the two-flavor quark model at zero temperature. In addition to reproducing the numerical results of our original short Letter [12], we also present many analytical results that have not been included in Ref. [12]. At zero temperature, the screening properties of gluons in the g2SC phase, as well as the possible appearance of two additional spin-1 condensates, are deduced from the qualitative nature of the low energy spectrum of quasiparticles. The finite temperature properties of the g2SC phase are studied in Sec. 4. Among main results, we find that (i) the phase transition between the g2SC phase and normal quark matter is a second order phase transition, (ii) the ratio of the critical temperature and the zero temperature gap is not a universal number in the gapless phase, and (iii) a nonzero finite temperature gap may appear even if its zero temperature value is vanishing. Sec. 5 contains our conclusions and a brief discussion of the properties of the g2SC phase as well as their applications. 


\section{Model}

\subsection{Lagrangian density and parameters of the model}

Let us start our analysis by discussing the quark model that we use in the following. We assume that the strange quark is sufficiently heavy and does not appear at intermediate baryon densities under consideration (e.g., this might correspond to the quark chemical potential $\mu \equiv \mu_{B} / 3$ in the range between about 350 and $450 \mathrm{MeV}$ ). Therefore, we use the simplest SU(2) Nambu-JonaLasinio (NJL) model that was proposed in Ref. [20]. The explicit form of the Lagrangian density reads:

$$
\begin{aligned}
L= & \bar{q}\left(i \gamma^{\mu} \partial_{\mu}-m_{0}\right) q+G_{S}\left[(\bar{q} q)^{2}+\left(\bar{q} i \gamma_{5} \vec{\tau} q\right)^{2}\right] \\
& +G_{D}\left[\left(i \bar{q}^{C} \varepsilon \epsilon^{b} \gamma_{5} q\right)\left(i \bar{q} \varepsilon \epsilon^{b} \gamma_{5} q^{C}\right)\right]
\end{aligned}
$$

where $q^{C}=C \bar{q}^{T}$ is the charge-conjugate spinor and $C=i \gamma^{2} \gamma^{0}$ is the charge conjugation matrix. The quark field $q \equiv q_{i \alpha}$ is a four-component Dirac spinor that carries flavor $(i=1,2)$ and color $(\alpha=1,2,3)$ indices. The Pauli matrices are denoted by $\vec{\tau}=\left(\tau^{1}, \tau^{2}, \tau^{3}\right)$, while $(\varepsilon)^{i k} \equiv \varepsilon^{i k}$ and $\left(\epsilon^{b}\right)^{\alpha \beta} \equiv \epsilon^{\alpha \beta b}$ are the antisymmetric tensors in the flavor and color spaces, respectively. We also introduce a momentum cutoff $\Lambda$, and two independent coupling constants in the scalar quark-antiquark and scalar diquark channels, $G_{S}$ and $G_{D}$.

The values of the parameters in the NJL model are chosen by fitting the pion decay constant $f_{\pi}=93 \mathrm{MeV}$ and the chiral condensate $\langle\bar{u} u\rangle^{1 / 3}=\langle\bar{d} d\rangle^{1 / 3}=$ $-250 \mathrm{MeV}$. In the case of $m_{0} \neq 0$, the additional parameter could also be fixed by fitting the value of the pion mass. In the rest of this paper, however, we consider only the chiral limit with $m_{0}=0$. Then the two model parameters, i.e., the coupling constant and the cutoff, are chosen as follows: $G_{S}=5.0163$ $\mathrm{GeV}^{-2}$ and $\Lambda=653.3 \mathrm{MeV}[20,21]$. Without loss of generality, we set the strength of the diquark coupling $G_{D}$ to be proportional to the quark-antiquark coupling constant, i.e., $G_{D}=\eta G_{S}$ with a typical number for $\eta$ being around $0.75[12]$.

When considering the chiral limit in the following, we shall find that the constituent quark mass is zero in the model at hand in the g2SC phase. In reality, however, this may not be the case. At the same time, since the appearance of the g2SC phase is a Fermi surface phenomenon, our assumption should not be a strong limitation. Of course, nonzero quark masses should affect the Fermi momenta of different quark species. In turn, this could change slightly the mismatch parameter $\delta \mu$ in our model (see below), and introduce some minor changes in the analysis [22]. However, to large extent, the results would not 
change.

\subsection{Thermodynamic potential in $\beta$-equilibrium}

In $\beta$-equilibrium, the diagonal matrix of quark chemical potentials is given in terms of quark, electrical and color chemical potentials,

$$
\mu_{i j, \alpha \beta}=\left(\mu \delta_{i j}-\mu_{e} Q_{i j}\right) \delta_{\alpha \beta}+\frac{2}{\sqrt{3}} \mu_{8} \delta_{i j}\left(T_{8}\right)_{\alpha \beta},
$$

where $Q$ and $T_{8}$ are generators of $\mathrm{U}(1)_{e m}$ of electromagnetism and the $\mathrm{U}(1)_{8}$ subgroup of the color gauge group. The explicit expressions for the quark chemical potentials read

$$
\begin{aligned}
\mu_{u r}=\mu_{u g} & =\mu-\frac{2}{3} \mu_{e}+\frac{1}{3} \mu_{8}, \\
\mu_{d r}=\mu_{d g} & =\mu+\frac{1}{3} \mu_{e}+\frac{1}{3} \mu_{8}, \\
\mu_{u b} & =\mu-\frac{2}{3} \mu_{e}-\frac{2}{3} \mu_{8}, \\
\mu_{d b} & =\mu+\frac{1}{3} \mu_{e}-\frac{2}{3} \mu_{8} .
\end{aligned}
$$

One should notice that, in general, there are two mutually commuting color charges related to the generators $T_{3}$ and $T_{8}$ of the $\mathrm{SU}(3)_{c}$ group. Therefore, one could introduce two chemical potentials for these two different color charges. However, we require that quark matter in the 2SC ground state remains invariant under the $\mathrm{SU}(2)_{c}$ color gauge subgroup. This condition makes the introduction of the second nontrivial color chemical potential $\mu_{3}$ unnecessary.

In the mean field approximation, the finite temperature effective potential for quark matter in $\beta$-equilibrium with electrons takes the form:

$$
\begin{aligned}
\Omega & =\Omega_{0}-\frac{1}{12 \pi^{2}}\left(\mu_{e}^{4}+2 \pi^{2} T^{2} \mu_{e}^{2}+\frac{7 \pi^{4}}{15} T^{4}\right)+\frac{\left(m-m_{0}\right)^{2}}{4 G_{S}} \\
& +\frac{\Delta^{2}}{4 G_{D}}-\sum_{a} \int \frac{d^{3} p}{(2 \pi)^{3}}\left[E_{a}+2 T \ln \left(1+e^{-E_{a} / T}\right)\right],
\end{aligned}
$$

where $\mu_{e}$ is the electron chemical potential and $\Omega_{0}$ is a constant added to make the pressure of the vacuum zero. For simplicity, we substituted the zero value of the electron mass which is sufficient for the purposes of the current study. The sum in the second line of Eq. (7) runs over all (6 quark and 6 antiquark) 
quasiparticles. The explicit dispersion relations and the degeneracy factors of the quasiparticles read

$$
\begin{aligned}
E_{u b}^{ \pm} & =E(p) \pm \mu_{u b}, & & {[\times 1] } \\
E_{d b}^{ \pm} & =E(p) \pm \mu_{d b}, & & {[\times 1] } \\
E_{\Delta^{ \pm}}^{ \pm} & =E_{\Delta}^{ \pm}(p) \pm \delta \mu . & & {[\times 2] }
\end{aligned}
$$

Here we introduced the following shorthand notation:

$$
\begin{aligned}
E(p) & \equiv \sqrt{\mathbf{p}^{2}+m^{2}}, \\
E_{\Delta}^{ \pm}(p) & \equiv \sqrt{[E(p) \pm \bar{\mu}]^{2}+\Delta^{2}}, \\
\bar{\mu} & \equiv \frac{\mu_{u r}+\mu_{d g}}{2}=\frac{\mu_{u g}+\mu_{d r}}{2}=\mu-\frac{\mu_{e}}{6}+\frac{\mu_{8}}{3}, \\
\delta \mu & \equiv \frac{\mu_{d g}-\mu_{u r}}{2}=\frac{\mu_{d r}-\mu_{u g}}{2}=\frac{\mu_{e}}{2} .
\end{aligned}
$$

The knowledge of the thermodynamic potential is sufficient to calculate all other thermodynamic properties of quark matter in equilibrium. In the model at hand, the physical thermodynamic potential that determines the pressure of quark matter, $\Omega_{\text {phys }}=-P$, is obtained from $\Omega$ in Eq. (7) after substituting $\mu_{8}$, $\mu_{e}, m$ and $\Delta$ that solve the color and electrical charge neutrality conditions, i.e.,

$$
n_{8} \equiv-\frac{\partial \Omega}{\partial \mu_{8}}=0, \quad \text { and } \quad n_{Q} \equiv-\frac{\partial \Omega}{\partial \mu_{e}}=0
$$

as well as the gap equations,

$$
\frac{\partial \Omega}{\partial m}=0, \quad \text { and } \quad \frac{\partial \Omega}{\partial \Delta}=0
$$

respectively. In the following two subsections, we shall derive the explicit expressions for the charge neutrality conditions and the gap equations.

\subsection{Electrical and color charge densities}

By enforcing only the charge neutrality conditions in Eq. (15), one can construct the effective potential of the neutral quark matter as a function of two order parameters $m$ and $\Delta$. The subsequent minimization of such a potential determines the physical values of the Dirac mass of quarks and the value of the superconducting gap in the ground state of neutral quark matter. 
From the potential in Eq. (7), we derive the expressions for the electrical charge density $n_{Q}$ and the color charge density $n_{8}$ by taking partial derivatives with respect to $\mu_{e}$ and $\mu_{8}$, respectively. As a result, we arrive at the following explicit expressions:

$$
\begin{aligned}
n_{8}= & \frac{4}{3} \int \frac{d^{3} \mathbf{p}}{(2 \pi)^{3}}\left[-\frac{E-\bar{\mu}}{E_{\Delta}^{-}}\left[1-\tilde{f}\left(E_{\Delta^{+}}^{-}\right)-\tilde{f}\left(E_{\Delta^{-}}^{-}\right)\right]\right. \\
& +\frac{E+\bar{\mu}}{E_{\Delta}^{+}}\left[1-\tilde{f}\left(E_{\Delta^{+}}^{+}\right)-\tilde{f}\left(E_{\Delta^{-}}^{+}\right)\right] \\
& \left.+\tilde{f}\left(E_{u b}^{+}\right)-\tilde{f}\left(E_{u b}^{-}\right)+\tilde{f}\left(E_{d b}^{+}\right)-\tilde{f}\left(E_{d b}^{-}\right)\right],
\end{aligned}
$$

and

$$
\begin{aligned}
n_{Q}= & -\frac{1}{2} n_{8}+\frac{\mu_{e}^{3}}{3 \pi^{2}}+\frac{1}{3} \mu_{e} T^{2}+2 \int \frac{d^{3} \mathbf{p}}{(2 \pi)^{3}}\left[\tilde{f}\left(E_{u b}^{+}\right)-\tilde{f}\left(E_{u b}^{-}\right)\right. \\
& \left.-\tilde{f}\left(E_{\Delta^{+}}^{-}\right)-\tilde{f}\left(E_{\Delta^{+}}^{+}\right)+\tilde{f}\left(E_{\Delta^{-}}^{+}\right)+\tilde{f}\left(E_{\Delta^{-}}^{-}\right)\right] .
\end{aligned}
$$

Here the standard Fermi distribution function has been introduced, $\tilde{f}(E)=$ $1 /[1+\exp (E / T)]$. From Eq. (18) we notice that the linear combination of charge densities $n_{Q}+\frac{1}{2} n_{8}$ is given by a rather simple expression. In this particular combination, the electrical and color charges of blue down quarks cancel. Had the dispersion relations in Eq. (10) been dependent on $\bar{\mu}$ only, the charge cancellation from the corresponding quasiparticles would also be exact. It is the presence of the mismatch parameter $\delta \mu$ that leads to a nonvanishing contribution. As we shall see, the latter survives even at zero temperature in the g2SC phase $(\delta \mu>\Delta)$, while disappear in the 2SC phase $(\delta \mu<\Delta)$.

In the following, we shall require both charge densities in Eqs. (17) and (18) to be zero. Such a requirement is necessary in order to have a realistic construction of dense quark matter that could appear in the bulk of a compact star. Indeed, without enforcing the neutrality, the repulsive Coulomb force would clearly overwhelm the gravity preventing the formation of a stable star.

Before concluding this subsection, we mention that it also makes sense to study quark matter at fixed values of chemical potentials without imposing the conditions of local neutrality. Such studies are particularly appropriate when globally neutral mixed phases are considered $[16,17]$. In this paper, however, we do not discuss mixed phases, but concentrate our attention exclusively on locally neutral (homogeneous) phases of quark matter in $\beta$-equilibrium. 


\subsection{Gap equations for order parameters}

Starting from the potential in Eq. (7), we derive an explicit form of the gap equations. In particular, the equation for the Dirac mass reads

$$
\begin{aligned}
m-m_{0}= & 4 G_{S} \int \frac{d^{3} \mathbf{p}}{(2 \pi)^{3}} \frac{m}{E}\left[2 \frac{E-\bar{\mu}}{E_{\Delta}^{-}}\left[1-\tilde{f}\left(E_{\Delta^{+}}^{-}\right)-\tilde{f}\left(E_{\Delta^{-}}^{-}\right)\right]\right. \\
& +2 \frac{E+\bar{\mu}}{E_{\Delta}^{+}}\left[1-\tilde{f}\left(E_{\Delta^{+}}^{+}\right)-\tilde{f}\left(E_{\Delta^{-}}^{+}\right)\right] \\
& \left.+2-\tilde{f}\left(E_{u b}^{+}\right)-\tilde{f}\left(E_{u b}^{-}\right)-\tilde{f}\left(E_{d b}^{+}\right)-\tilde{f}\left(E_{d b}^{-}\right)\right]
\end{aligned}
$$

In the chiral limit $\left(m_{0}=0\right)$, the trivial solution $m=0$ corresponds to a chirally symmetric phase of quark matter, while a nontrivial solution $m \neq 0$ corresponds to a phase with spontaneously broken chiral symmetry.

Similarly, we derive the gap equation for the diquark condensate,

$$
\begin{aligned}
\Delta= & 8 G_{D} \int \frac{d^{3} \mathbf{p}}{(2 \pi)^{3}}\left[\frac{\Delta}{E_{\Delta}^{-}}\left[1-\tilde{f}\left(E_{\Delta^{+}}^{-}\right)-\tilde{f}\left(E_{\Delta^{-}}^{-}\right)\right]\right. \\
& \left.+\frac{\Delta}{E_{\Delta}^{+}}\left[1-\tilde{f}\left(E_{\Delta^{+}}^{+}\right)-\tilde{f}\left(E_{\Delta^{-}}^{+}\right)\right]\right] .
\end{aligned}
$$

This equation can also have trivial as well as nontrivial solutions for $\Delta$. In the latter case, the corresponding ground state is a color superconductor.

\subsection{Number densities of quarks}

According to one of the criteria of the g2SC phase, the densities of the quark species that participate in pairing dynamics are not equal at zero temperature [12]. This is in contrast to regular pairing in the conventional gaped color superconductors [19].

Even before studying the effect of the Cooper pairing on the quark densities, we should discuss how to derive the formal expressions for these densities. One appreciates immediately, of course, that the definitions of such densities are unambiguous only if the chemical potentials of all six types (2 flavors times 3 colors) of quarks are treated as independent parameters. This is exactly how we proceed. The quark densities are obtained by taking the partial derivatives of the potential $\Omega$ in Eq. (7) with respect to these independent parameters. At the end, the relations between the chemical potentials required by the 
$\beta$-equilibrium are imposed. The only point that needs some additional explanation is how to interpret the dependence of the thermodynamic potential in Eq. (7) on the parameters $\bar{\mu}$ and $\delta \mu$, entering through the quasiparticle energies in Eq. (10). It turns out that $\bar{\mu}$ and $\delta \mu$ should be replaced by $\frac{1}{2}\left(\mu_{d g}+\mu_{u r}\right)$ and $\frac{1}{2}\left(\mu_{d g}-\mu_{u r}\right)$, respectively, in one set of the double degenerate modes, and by $\frac{1}{2}\left(\mu_{d r}+\mu_{u g}\right)$ and $\frac{1}{2}\left(\mu_{d r}-\mu_{u g}\right)$ in the other set, see Eqs. (13) and (14).

Because of the residual $S U(2)_{c}$ symmetry in the ground state of quark matter, the densities of the same flavor, red and green quarks are equal in the g2SC ground state. For example, the densities of the up quarks participating in the Cooper pairing read

$$
\begin{aligned}
n_{u r}=n_{u g}= & \int \frac{d^{3} \mathbf{p}}{(2 \pi)^{3}}\left[\frac{E+\bar{\mu}}{E_{\Delta}^{+}}\left[1-\tilde{f}\left(E_{\Delta^{+}}^{+}\right)-\tilde{f}\left(E_{\Delta^{-}}^{+}\right)\right]\right. \\
& -\frac{E-\bar{\mu}}{E_{\Delta}^{-}}\left[1-\tilde{f}\left(E_{\Delta^{+}}^{-}\right)-\tilde{f}\left(E_{\Delta^{-}}^{-}\right)\right] \\
& \left.+\tilde{f}\left(E_{\Delta^{+}}^{-}\right)+\tilde{f}\left(E_{\Delta^{+}}^{+}\right)-\tilde{f}\left(E_{\Delta^{-}}^{+}\right)-\tilde{f}\left(E_{\Delta^{-}}^{-}\right)\right] .
\end{aligned}
$$

Similarly, for the densities of the down quarks, we get

$$
\begin{aligned}
n_{d g}=n_{d r}= & \int \frac{d^{3} \mathbf{p}}{(2 \pi)^{3}}\left[\frac{E+\bar{\mu}}{E_{\Delta}^{+}}\left[1-\tilde{f}\left(E_{\Delta^{+}}^{+}\right)-\tilde{f}\left(E_{\Delta^{-}}^{+}\right)\right]\right. \\
& -\frac{E-\bar{\mu}}{E_{\Delta}^{-}}\left[1-\tilde{f}\left(E_{\Delta^{+}}^{-}\right)-\tilde{f}\left(E_{\Delta^{-}}^{-}\right)\right] \\
& \left.-\tilde{f}\left(E_{\Delta^{+}}^{-}\right)-\tilde{f}\left(E_{\Delta^{+}}^{+}\right)+\tilde{f}\left(E_{\Delta^{-}}^{+}\right)+\tilde{f}\left(E_{\Delta^{-}}^{-}\right)\right] .
\end{aligned}
$$

As we see, the densities of the up and down quarks participating in the Cooper pairing are not equal. In fact, the difference of the densities is given by

$$
\begin{aligned}
n_{d g}-n_{u r} & =n_{d r}-n_{u g} \\
& =2 \int \frac{d^{3} \mathbf{p}}{(2 \pi)^{3}}\left[\tilde{f}\left(E_{\Delta^{-}}^{-}\right)-\tilde{f}\left(E_{\Delta^{+}}^{-}\right)+\tilde{f}\left(E_{\Delta^{-}}^{+}\right)-\tilde{f}\left(E_{\Delta^{+}}^{+}\right)\right],
\end{aligned}
$$

which is always nonzero at finite temperature provided the mismatch parameter $\delta \mu$ is nonzero. It is even more important for us here that this difference is nonzero at zero temperature in the g2SC phase of quark matter,

$$
\begin{aligned}
\left.\left(n_{d g}-n_{u r}\right)\right|_{T=0} & =\left.\left(n_{d r}-n_{u g}\right)\right|_{T=0} \\
& =\theta(\delta \mu-\Delta) \frac{2}{3 \pi^{2}} \sqrt{(\delta \mu)^{2}-\Delta^{2}}\left(3 \bar{\mu}^{2}+(\delta \mu)^{2}-\Delta^{2}\right) .
\end{aligned}
$$


This is in contrast to the 2SC phase $(\delta \mu<\Delta)$ where this difference is zero in agreement with the arguments of Ref. [19].

The unpaired blue quarks, $u_{b}$ and $d_{b}$, are singlet states with respect to $\mathrm{SU}(2)_{c}$ symmetry of the ground state. The densities of these quarks are

$$
n_{u b}=2 \int \frac{d^{3} \mathbf{p}}{(2 \pi)^{3}}\left[\tilde{f}\left(E_{u b}^{-}\right)-\tilde{f}\left(E_{u b}^{+}\right)\right] \simeq \frac{\mu_{u b}}{3}\left(\frac{\mu_{u b}^{2}}{\pi^{2}}+T^{2}\right),
$$

for the up quarks, and

$$
n_{d b}=2 \int \frac{d^{3} \mathbf{p}}{(2 \pi)^{3}}\left[\tilde{f}\left(E_{d b}^{-}\right)-\tilde{f}\left(E_{d b}^{+}\right)\right] \simeq \frac{\mu_{d b}}{3}\left(\frac{\mu_{d b}^{2}}{\pi^{2}}+T^{2}\right),
$$

for the down quarks, respectively.

\section{Gapless 2SC at zero temperature}

Here we present a detailed study of the zero temperature properties of dense two-flavor quark matter. A brief outline of this study was presented in Ref. [12]. In this paper, we include additionally many new results (e.g., regarding the gluon properties in g2SC phase of quark matter, and the appearance of spin-1 color superconducting gaps on top of the g2SC phase) that were outside the scope of the original publication [12].

At zero temperature, the effective potential (7) for quark matter in $\beta$-equilibrium with electrons takes the following simple form [12]:

$$
\Omega=\Omega_{0}-\frac{\mu_{e}^{4}}{12 \pi^{2}}+\frac{m^{2}}{4 G_{S}}+\frac{\Delta^{2}}{4 G_{D}}-\sum_{a} \int \frac{d^{3} p}{(2 \pi)^{3}}\left|E_{a}\right|
$$

Here, without loss of generality, we assume that $m_{0}=0$ (chiral limit). In this case, the constituent quark mass is also zero in the color superconducting phase

of matter at sufficiently large baryon chemical potential. Thus, we substitute $m=0$ below. After making use of the quasiparticle energies in Eqs. (8)-(10), we derive

$$
\begin{array}{r}
\Omega=\Omega_{0}-\frac{\mu_{e}^{4}}{12 \pi^{2}}+\frac{\Delta^{2}}{4 G_{D}}-\frac{\Lambda^{4}}{2 \pi^{2}}-\frac{\mu_{u b}^{4}}{12 \pi^{2}}-\frac{\mu_{d b}^{4}}{12 \pi^{2}} \\
-2 \int_{0}^{\Lambda} \frac{p^{2} d p}{\pi^{2}}\left(\sqrt{(p+\bar{\mu})^{2}+\Delta^{2}}+\sqrt{(p-\bar{\mu})^{2}+\Delta^{2}}\right)
\end{array}
$$




$$
-2 \theta(\delta \mu-\Delta) \int_{\mu^{-}}^{\mu^{+}} \frac{p^{2} d p}{\pi^{2}}\left(\delta \mu-\sqrt{(p-\bar{\mu})^{2}+\Delta^{2}}\right)
$$

where $\mu^{ \pm} \equiv \bar{\mu} \pm \sqrt{(\delta \mu)^{2}-\Delta^{2}}$. The appearance of the last term that contains the step function $\theta(\delta \mu-\Delta)$ is remarkable. It can be traced back to the existence of two degenerate gapless modes in the quasiparticle spectrum, whose energies change sign at the values of momenta $p=\mu^{ \pm}$, see Eq. (10). In a different language, this sign change modifies the prescription for treating the quasiparticle poles in the complex energy plane. This is exactly the reason why the arguments of Ref. [19] regarding the conventional "enforced" pairing in color superconductors do not apply in the case of g2SC. The nonequal densities of pairing quarks, see Eq. (24), is the formal consequence of this fact.

\subsection{Solving neutrality conditions}

Now, let us proceed to the analysis of the color and electrical charge neutrality conditions in the model of dense quark matter at hand. At zero temperature, the corresponding two independent conditions could be written in the form: $n_{8}=0$ and $n_{Q}+\frac{1}{2} n_{8}=0$, i.e.,

$$
\begin{aligned}
n_{8}= & \frac{4}{3} \int \frac{d^{3} \mathbf{p}}{(2 \pi)^{3}}\left(-\frac{E-\bar{\mu}}{E_{\Delta}^{-}} \theta\left(E_{\Delta^{-}}^{-}\right)+\frac{E+\bar{\mu}}{E_{\Delta}^{+}}-\theta\left(-E_{u b}^{-}\right)-\theta\left(-E_{d b}^{-}\right)\right) \\
\simeq & \frac{2}{9 \pi^{2}}\left(2 \bar{\mu}^{3}-\mu_{u b}^{3}-\mu_{d b}^{3}\right)+\frac{2 \bar{\mu} \Delta^{2}}{3 \pi^{2}}\left(\ln \frac{4\left(\Lambda^{2}-\bar{\mu}^{2}\right)}{\Delta^{2}}-\frac{2 \Lambda^{2}-\bar{\mu}^{2}}{\Lambda^{2}-\bar{\mu}^{2}}+\cdots\right) \\
& +\frac{2 \bar{\mu}}{3 \pi^{2}} \theta(\delta \mu-\Delta)\left(2 \delta \mu \sqrt{(\delta \mu)^{2}-\Delta^{2}}-\Delta^{2} \ln \frac{\delta \mu+\sqrt{(\delta \mu)^{2}-\Delta^{2}}}{\delta \mu-\sqrt{(\delta \mu)^{2}-\Delta^{2}}}\right) \\
= & 0
\end{aligned}
$$

where the ellipsis denotes the terms of higher order in powers of $\Delta$, and

$$
\begin{aligned}
n_{Q}+\frac{1}{2} n_{8} & =\frac{\mu_{e}^{3}}{3 \pi^{2}}+2 \int \frac{d^{3} \mathbf{p}}{(2 \pi)^{3}}\left(-\theta\left(-E_{u b}^{-}\right)+\theta\left(-E_{\Delta^{-}}^{-}\right)\right) \\
& =\frac{\mu_{e}^{3}-\mu_{u b}^{3}}{3 \pi^{2}}+\frac{2}{3 \pi^{2}} \theta(\delta \mu-\Delta) \sqrt{(\delta \mu)^{2}-\Delta^{2}}\left(3 \bar{\mu}^{2}+(\delta \mu)^{2}-\Delta^{2}\right) \\
& =0 .
\end{aligned}
$$




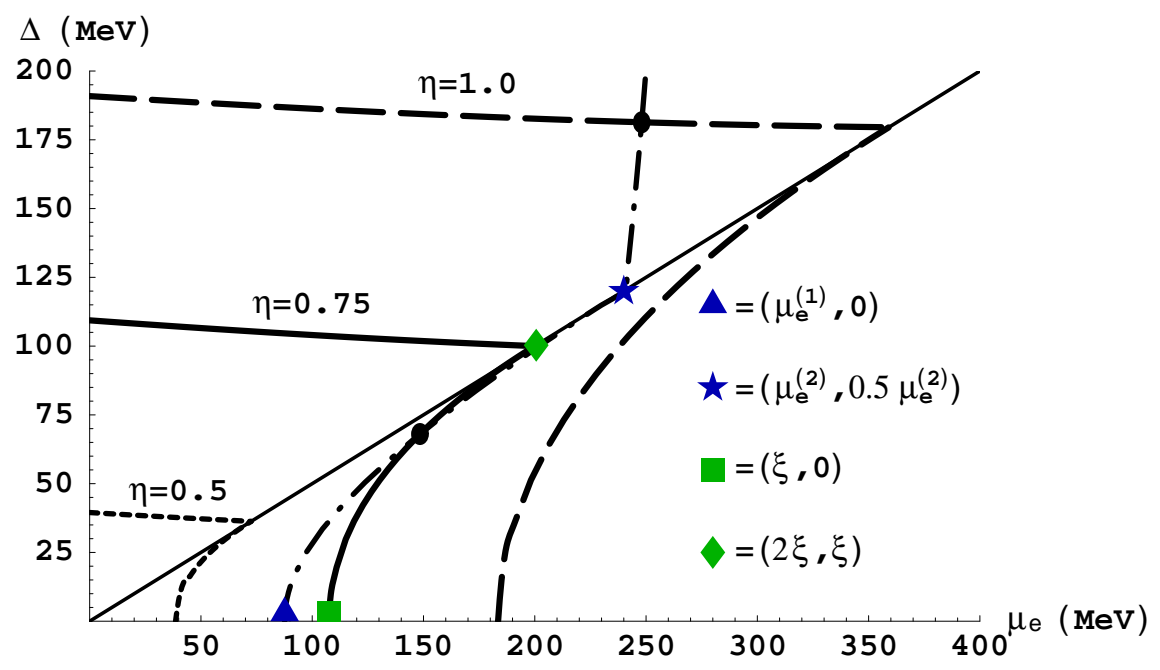

Fig. 1. The graphical representation of the solution to the charge neutrality conditions (thick dash-dotted line) and the solution to the gap equation for three different values of the diquark coupling constant (thick solid and dashed lines). The intersection points represent the solutions to both. The thin solid line divides two qualitatively different regions, $\Delta<\delta \mu$ and $\Delta>\delta \mu$. The results are plotted for $\mu=400 \mathrm{MeV}$ and three values of diquark coupling constant $G_{D}=\eta G_{S}$ with $\eta=0.5, \eta=0.75$, and $\eta=1.0$.

This last condition is particularly simple in the $2 \mathrm{SC}$ phase, $\Delta \geq \delta \mu$. In this case, the exact solution to this equation is available:

$$
\mu_{e}=\frac{3 \mu-2 \mu_{8}}{5}, \quad \text { for } \quad \Delta \geq \delta \mu .
$$

The other equation, then, has the following approximate solution:

$$
\mu_{8} \simeq \frac{45}{44}\left(\frac{4}{5}-6^{1 / 3}-\frac{4^{1 / 3}}{3^{1 / 3}}\right) \mu+\cdots \approx 0.0854 \mu+\cdots,
$$

where the ellipsis again denotes the terms of higher order in powers of $\Delta / \mu$. As we see, the color chemical potential $\mu_{8}$ is very small compared to the other chemical potentials, $\mu_{e}$ and $\mu$. Of course, the corrections to the above solutions may become large with increasing the value of the gap $\Delta$. Therefore, strictly speaking, these formulas are reliable only in the weakly coupled regime when $\Delta \ll \mu$. Our numerical results show, however, that $\mu_{8}$ remains also small at relatively large values of the gap. Moreover, the approximation $\mu_{8} \approx 0$ works well in the region $\Delta<\delta \mu$ and in a wide range of parameters in the model. In the following, we shall use this observation to derive many useful analytical results. 
Now, let us consider the neutrality condition in the region $\Delta \leq \delta \mu$. In our analytical treatment, we substitute $\mu_{8}=0$. As we mentioned above, this is a very good approximation. In the case of small values of $\Delta$ this should not be surprizing since the color charge vanishes in the normal phase when $\mu_{8}=0$. The value of the electrical chemical potential is determined by solving the neutrality condition in Eq. (30). The corresponding result for $\mu_{e}^{\left(n_{Q}=0\right)}(\mu, \Delta)$ could be given by the inverse representation of the gap in terms of the potential $\mu_{e}$,

$$
\Delta^{\left(n_{Q}=0\right)}=\sqrt{\frac{\mu_{e}^{2}}{4}-\left(\frac{\left(\mu-2 \mu_{e} / 3\right)^{3}-\mu_{e}^{3}}{6\left(\mu-\mu_{e} / 6\right)^{2}}\right)^{2}}, \quad \text { for } \quad \Delta \leq \delta \mu .
$$

This analytical result is barely distinguishable from the numerical solution represented by the dash-dotted line in Fig. 1. Quark matter is positively charged to the left from the neutrality line, and it is negatively charged to the right.

From Eq. (33), we find an approximate point of the intersection of the neutrality line with the $\mu_{e}$-axis $(\Delta=0)$. This is denoted by a triangle in Fig. 1 . This point determines the value of the electrical chemical potential in the normal phase of neutral quark matter. Its approximate value is given by a solution to a cubic equation, obtained from Eq. (33) after substituting $\Delta=0$ on the left hand side. This value is $\mu_{e}^{(1)} \simeq 0.2196 \mu$. For example, at $\mu=400 \mathrm{MeV}$, it is $87.84 \mathrm{MeV}$ which is in good agreement with the numerical result plotted in Fig. 1.

Also, from Eq. (33), we determine the intersection of the neutrality line with the line $\Delta=\delta \mu$ that separates two qualitatively different regions in the plane $\left(\mu_{e}, \Delta\right)$. This occurs at $\mu_{e}^{(2)} \simeq \frac{3}{5} \mu$ that corresponds to $\Delta^{(2)}=\frac{1}{2} \mu_{e}^{(2)} \simeq \frac{3}{10} \mu$. For the choice of the quark chemical potential $\mu=400 \mathrm{MeV}$, used in Fig. 1, the corresponding values are $\mu_{e}^{(2)} \simeq 240 \mathrm{MeV}$ and $\Delta^{(2)} \simeq 120 \mathrm{MeV}$ which are again in good agreement with the numerical result (cf., the location of the "knee" of the dash-dotted line in Fig. 1, denoted by a star).

Before concluding this subsection, we should emphasize that the charge densities in Eqs. (29) and (30) do not depend explicitly on the diquark coupling constant. This is the property of the mean field approximation that would break after taking higher order corrections into account. It is reasonable to assume that the corresponding dependence is weak even in the full theory. As we shall see below, this property is not shared by the solution for the gap $\Delta$ which is very sensitive to changes in the coupling constant. This observation would have far reaching consequences below. 


\subsection{Solving gap equation}

Now, we turn to the solution of the gap equation (20) at zero temperature. In this case, the equation simplifies considerably. Its explicit form reads

$$
\begin{aligned}
& \Delta=8 G_{D} \int \frac{d^{3} \mathbf{p}}{(2 \pi)^{3}}\left(\frac{\Delta}{E_{\Delta}^{-}} \theta\left(E_{\Delta^{-}}^{-}\right)+\frac{\Delta}{E_{\Delta}^{+}}\right) \simeq \frac{4 G_{D} \Delta}{\pi^{2}}\left[\Lambda^{2}-3 \bar{\mu}^{2}\right. \\
& +\left(\bar{\mu}^{2}-\frac{1}{2} \Delta^{2}\right) \ln \frac{4\left(\Lambda^{2}-\bar{\mu}^{2}\right)}{\Delta^{2}}+\frac{\Delta^{2}\left(\Lambda^{4}+3 \Lambda^{2} \bar{\mu}^{2}-2 \bar{\mu}^{2}\right)}{2\left(\Lambda^{2}-\bar{\mu}^{2}\right)^{2}}+\cdots \\
& \left.-\theta(\delta \mu-\Delta)\left(\delta \mu \sqrt{(\delta \mu)^{2}-\Delta^{2}}+\left(\bar{\mu}^{2}-\frac{1}{2} \Delta^{2}\right) \ln \frac{\delta \mu+\sqrt{(\delta \mu)^{2}-\Delta^{2}}}{\delta \mu-\sqrt{(\delta \mu)^{2}-\Delta^{2}}}\right)\right] .
\end{aligned}
$$

As it should be, this equation always has the trivial solution $\Delta=0$. This corresponds to the normal phase of quark matter. If there is also a nontrivial solution $\Delta \neq 0$, the normal phase is not necessarily the ground state. In fact, the true ground state corresponds to the solution that minimizes the value of the thermodynamic potential (i.e., it maximizes the pressure). Moreover, in the case of an additional requirement of neutrality, the minimization should be done along the neutrality line discussed in the preceding subsection.

For a moment, let us drop the condition of the charge neutrality and consider all possible nontrivial solutions to the gap equation (34). We realize right away that there are two different branches of solutions in two regions, $\Delta<\delta \mu$ and $\Delta>\delta \mu$, see Fig. 1 .

First, we start with the region $\Delta>\delta \mu$. This is the case of a relatively small mismatch between the Fermi momenta of the pairing quarks. In particular, it describes the conventional 2SC phase of quark matter. From Eq. (34), we derive the approximate analytical expression for the nontrivial solution,

$$
\Delta \simeq \xi(\bar{\mu}), \quad \text { for } \quad \Delta>\delta \mu
$$

where

$$
\xi(\bar{\mu}) \equiv 2 \sqrt{\Lambda^{2}-\bar{\mu}^{2}} \exp \left(-\frac{\pi^{2}}{8 \bar{\mu}^{2} G_{D}}+\frac{\Lambda^{2}-3 \bar{\mu}^{2}}{2 \bar{\mu}^{2}}\right) .
$$

Since the average chemical potential $\bar{\mu}$ depends on $\mu_{e}$, see Eq. (13), the above solution for the gap is also a function of $\mu_{e}$. This dependence, however, is very weak because $\mu_{e}$ enters $\bar{\mu}$ with a relatively small weight. This is also confirmed by our numerical results for three different values of the diquark coupling constant presented in Fig. 1 . With increasing $\mu_{e}$, the value of the gap 
decreases by less than $10 \%$ along the whole upper branch of the solution. In contrast, the dependence of the gap on the coupling constant is very sensitive.

In the region $\Delta<\delta \mu$, the mismatch parameter $\delta \mu$ is large. This is where the g2SC phase of quark matter appears. The solution to the gap equation in this region is approximated well by the following expression:

$$
\Delta \simeq \sqrt{\xi\left(\mu_{e}-\xi\right)}, \quad \text { for } \quad \Delta<\delta \mu
$$

where $\xi$ is the same weakly dependent function of $\mu_{e}$ that was defined in Eq. (36). By making use of this analytical expression, we see that the lower branch of the solution to the gap equation extends only over a finite range of chemical potentials, $\mu_{e}^{(\min )} \leq \mu_{e} \leq \mu_{e}^{(\max )}$ (see Fig. 1). The values of $\mu_{e}^{(\min )}$ and $\mu_{e}^{(\max )}$ are determined by the solutions to the equations $\mu_{e}=\xi\left(\mu_{e}\right)$ and $\mu_{e}=$ $2 \xi\left(\mu_{e}\right)$. The corresponding graphical solutions are represented by the square and the diamond in Fig. 1 , respectively. At $\mu=400 \mathrm{MeV}$ and $\eta=0.75$, the corresponding approximate values are $\mu_{e}^{(\min )} \approx 107.9 \mathrm{MeV}$ and $\mu_{e}^{(\max )} \approx 205.3$ $\mathrm{MeV}$ which are in reasonable agreement with numerical results in Fig. 1.

In summary of this subsection, we note that the general nontrivial solution to the gap equation consists of two different branches in two regions, $\Delta>\delta \mu$ and $\Delta<\delta \mu$. The upper is the main branch that extends down to $\mu_{e}=0$ (i.e., the case without a mismatch in the Fermi surfaces of the up and down quarks). The lower branch appears only in a finite window of electrical chemical potentials. It merges with the upper one at a point on the line $\Delta=\delta \mu$. In Fig. 1, three solutions to the gap equation at $\mu=400 \mathrm{MeV}$ in three regimes with different coupling constants, corresponding to $\eta=0.5, \eta=0.75$, and $\eta=1.0$, are shown. The three curves have qualitatively the same shape, but differ by overall scale factors. As we shall see below, this difference in the overall scales has an important physical consequence. In particular, at weak coupling $(\eta \lesssim 0.7)$, the ground state of neutral quark matter is the normal phase, while neutral g2SC and 2SC phases are most stable at intermediate $(0.7 \lesssim \eta \lesssim 0.8)$ and strong $(\eta \gtrsim 0.8)$ couplings, respectively. This is studied in detail in the following subsection.

\subsection{Ground state of neutral matter}

The ground state of neutral matter is determined by the location of the minimum of the effective potential as a function of the gap. It is understood that the minimization is performed after the neutrality condition is fulfilled. In different words, the consideration is restricted only to the neutrality (dash-dotted) 
line in Fig. 1. Mathematically, the necessary condition for the minimum reads

$$
\left(\frac{d \Omega}{d \Delta}\right)_{n_{Q}, n_{8}=0} \equiv \frac{\partial \Omega}{\partial \Delta}+\frac{\partial \Omega}{\partial \mu_{e}}\left(\frac{d \mu_{e}}{d \Delta}\right)_{n_{Q}, n_{8}=0}+\frac{\partial \Omega}{\partial \mu_{8}}\left(\frac{d \mu_{8}}{d \Delta}\right)_{n_{Q}, n_{8}=0}=0
$$

We notice that the last two terms with the derivatives taken along the neutrality line are proportional to the electrical and color charge densities. They become zero when the requirement of neutrality is enforced. Therefore, the above condition formally coincides with the gap equation studied in the preceding subsection. The important difference is that Eq. (38) is defined only at the neutrality line in the $\left(\mu_{e}, \Delta\right)$ plane.

In order to satisfy the condition in Eq. (38), it is very convenient to use a simple graphical construction, presented in Fig. 1. The extremum values of the gap are determined by the points of intersection of the neutrality (dashdotted) line with the solutions to the gap equation (i.e., either with one of the branches of the non-trivial solution, or with $\Delta=0$ line).

At very weak coupling (e.g., $\eta=0.5$ case), both branches of the nontrivial solutions to the gap equation are squeezed into a small region around the origin in the $\left(\mu_{e}, \Delta\right)$ plane. As a result, the neutrality line does not intersect them. Therefore, the only extremum of the effective potential corresponds to the trivial solution $\Delta=0$. As is easy to see, this is the global minimum. Thus, the normal phase is the ground state of quark matter in this regime.

In the opposite case of very strong coupling (e.g., $\eta=1$ case), the potential has two extrema. Of course, one of them corresponds to the normal phase $(\Delta=0)$. This is a local maximum of the potential. The other extremum is a minimum that corresponds to the 2SC phase with $\Delta>\delta \mu$. Note that the types of the extrema could be inferred from the expected topology of the effective potential after taking into account that this potential is bounded below when $\Delta \rightarrow \infty$. Our direct calculation show that the 2SC phase is indeed the ground state. At large coupling, this is hardly a surprise.

The regime of intermediate coupling (see, e.g., $\eta=0.75$ case) is most interesting. At first sight, it looks similar to the case of strong coupling because the potential has two extrema. One of them occurs at $\Delta=0$ and the other at $\Delta \neq 0$. The peculiar thing is that the nontrivial extremum corresponds to an intersection of the neutrality line with the lower branch (defined in the region $\Delta<\delta \mu$ ) of the nontrivial solutions to the gap equation. By making use of the same topological arguments as in the previous case, we conclude that the nontrivial solution defines the ground state of neutral quark matter. This can also be checked by a direct numerical calculation of the effective potential, see the solid line in Fig. 2. As we shall discuss later in more detail, this ground state with $\Delta \neq 0$ is the g2SC, rather than ordinary 2SC phase. 


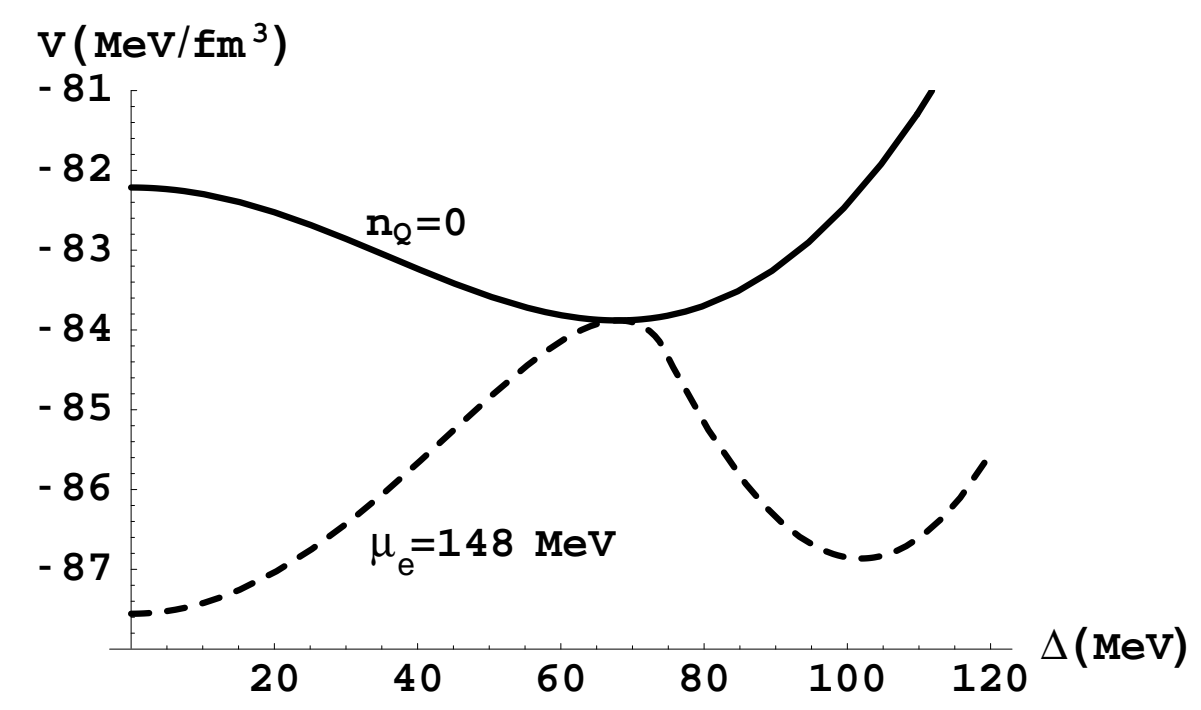

Fig. 2. The effective potential as a function of the diquark gap $\Delta$ calculated at a fixed value of the electrical chemical potential $\mu_{e}=148.4 \mathrm{MeV}$ (dashed line), and the effective potential defined along the neutrality line (solid line). The results are plotted for $\mu=400 \mathrm{MeV}$ and $G_{D}=\eta G_{S}$ with $\eta=0.75$.

At this point, it is relevant to make a few comments regarding the early studies of the color superconducting quark matter at finite values of the electrical (or, equivalently, isospin) chemical potential [23]. There, the electrical chemical potential was treated as a free parameter, and the charge neutrality condition was not imposed. Obviously, the 2SC phase is the ground state of quark matter at small values of the mismatch parameter $\delta \mu$ (which is identical with $\mu_{e} / 2$ here). However, when the value of $\delta \mu$ increases and reaches a critical value, a first order phase transition happens. The approximate value of the critical electrical chemical potential is $\mu_{e}^{\mathrm{cr}} \simeq \sqrt{2} \Delta_{0}$ where $\Delta_{0}$ is the value of the gap at $\mu_{e}=0$ (strictly speaking, this estimate is derived in the approximation of weak coupling [24]). At higher values of $\delta \mu$, the normal phase is the new ground state of quark matter. In this picture, there is no room for the g2SC phase.

Now, how can one reconcile the appearance of the g2SC phase after imposing the charge neutrality conditions, and its absence without such conditions? To answer this, we refer again to Fig. 1. In absence of the neutrality condition, one defines the potential $V(\Delta)$ by restricting $\Omega\left(\Delta, \mu_{e}\right)$ to a vertical line $\mu_{e}=$ Const for each fixed value of the parameter $\mu_{e}$. As a result, one ends up with a whole family of the effective potentials that are functions of $\Delta$. By changing the parameter $\mu_{e}$, one goes from one representative of the family to another. The extrema of the effective potentials are determined by the intersection points of $\mu_{e}=$ Const line with the line of solutions to the gap equation. 
Sweeping the values of $\mu_{e}$ from left to right in Fig. 1, one easily understands the reason for the first order phase transition in the quark matter. At small $\mu_{e}$, the potential has two extrema at $\Delta=0$ and at $\Delta \neq 0$. Naturally, the latter is the ground state. At intermediate values of $\mu_{e}$ where the lower branch of

the solution to the gap equation exists, $\mu_{e}^{(\min )}<\mu_{e}<\mu_{e}^{(\max )}$, the potential has already three extrema that are given by the intersection of the line $\mu_{e}=$ Const with the $\mu_{e}$-axis (i.e., $\Delta=0$ solution) and with the two branches of the nontrivial solutions. Interestingly, the solution on the lower branch corresponds to a maximum of the potential. The same solution in the neutral matter was the global minimum of the properly defined potential, see Fig. 2. The other two extrema are minima. It is the competition between them, that results in a first order transition from the 2SC to the normal phase.

It should be clear that this first order phase transition is unphysical under the requirement of local neutrality of quark matter. This is because the transition typically happens between two types of matter with different, in general, nonzero charge densities (e.g., positively charged color superconducting matter and negatively charged normal quark matter). It is worth mentioning, however, that this first order phase transition can get physical meaning in globally neutral mixed phases of quark matter [16,17], and in some condensed matter systems where there is no analogue of the charge neutrality condition [25].

\subsection{Weak, intermediate and strong coupling regimes}

Let us discuss the rigorous meaning of the weak, intermediate and strong coupling regimes in the model at hand (as we shall see below, this could get somewhat ambiguous at finite temperature). We start from the weak regime first. By definition, the normal phase is the only neutral phase in this case. As should be clear, this happens when there is no intersection of the neutrality line with the two nontrivial branches of the solution to the gap equation.

From Eq. (33) and the discussion following it, we know that the neutrality line intersects the $\mu_{e}$-axis at $\mu_{e}=\mu_{e}^{(1)} \approx 0.22 \mu$ (the corresponding point is marked by a triangle in Fig. 1). The lower branch of the gap solution, on the other hand, starts at $\mu_{e}=\xi$, see Eq. (37) and the point marked by a square in Fig. 1. Therefore, these two lines do not intersect if $\xi<\mu_{e}^{(1)}$, and only the normal phase is consistent with the condition of neutrality, see the $\eta=0.5$ case in Fig. 1. Now, we remind that while $\mu_{e}^{(1)}$ is essentially independent of the coupling constant, the value of $\xi$, for the definition see Eq. (36), is very sensitive to the choice of the diquark coupling. Taking this into account, we 
derive the following approximate condition of the weakly coupled regime:

$$
G_{D}<G_{D}^{(1)} \simeq \frac{\pi^{2}}{4 \Lambda^{2}-12 \bar{\mu}_{1}^{2}+4 \bar{\mu}_{1}^{2} \ln \left[4\left(\Lambda^{2}-\bar{\mu}_{1}^{2}\right) /\left(\mu_{e}^{(1)}\right)^{2}\right]}, \quad \text { "weak", }
$$

with $\bar{\mu}_{1} \simeq \mu-\frac{1}{6} \mu_{e}^{(1)} \approx 0.96 \mu$ here. At $\mu=400 \mathrm{MeV}$, the above estimate gives $\eta \lesssim 0.684$.

At larger coupling, the ground state of neutral matter is a broken phase. However, there are still two qualitatively different regimes depending on the location of the intersection of the neutrality line with the gap solution. If this occurs in the region $\Delta<\delta \mu$, the g2SC phase is the ground state (e.g., $\eta=0.75$ case in Fig. 1). Otherwise, the ground state is $2 \mathrm{SC}$ (e.g., $\eta=1$ case in Fig. 1).

The criterion for the strong coupling regime reads $2 \xi>\mu_{e}^{(2)}=0.6 \mu$, i.e., $\xi>0.3 \mu$. To derive this, we note that the value of the electrical chemical potential $\mu_{e}=0.6 \mu$ corresponds to the intersection of the neutrality line with the boundary $\Delta=\delta \mu$ (this is marked by a star in Fig. 1). If this value is smaller than $\mu_{e}=2 \xi$ at which the gap solution intersects the boundary line (this is marked by a diamond in Fig. 1), then the ground state is the 2SC phase. This is satisfied if the value of the coupling strength in the diquark channel is sufficiently strong, i.e.,

$$
G_{D}>G_{D}^{(2)} \simeq \frac{\pi^{2}}{4 \Lambda^{2}-12 \bar{\mu}_{2}^{2}+4 \bar{\mu}_{2}^{2} \ln \left[4\left(\Lambda^{2}-\bar{\mu}_{2}^{2}\right) /\left(\mu_{e}^{(2)} / 2\right)^{2}\right]}, \quad \text { "strong", }
$$

with $\bar{\mu}_{2} \simeq \mu-\frac{1}{6} \mu_{e}^{(2)}=0.9 \mu$ here. At $\mu=400 \mathrm{MeV}$, this estimate gives $\eta \gtrsim 0.806$.

Finally, the case of intermediate coupling strength is realized when $G_{D}^{(1)} \leq$ $G_{D} \leq G_{D}^{(2)}$, or alternatively, $\mu_{e}^{(1)} \simeq 0.22 \mu \lesssim \xi \lesssim 0.3 \mu \simeq \mu_{e}^{(2)} / 2$. In this case, the ground state is the g2SC phase. We check that, at $\mu=400 \mathrm{MeV}$, this is realized when $0.684 \lesssim \eta \lesssim 0.806$. A representative solution in this regime (with $\eta=0.75$ ) is shown in Fig. 1 by the thick solid line.

We would like to mention that the critical values of the coupling constant $G_{D}^{(1)}$ and $G_{D}^{(2)}$ in Eqs. (39) and (40) are functions of the quark chemical potential $\mu$, although the corresponding dependence is weak. Nevertheless, the above qualitative picture remains the same independent of the actual value of the quark chemical potential. Namely, there are always two critical values of the coupling constant that separate the three qualitatively different regimes. 

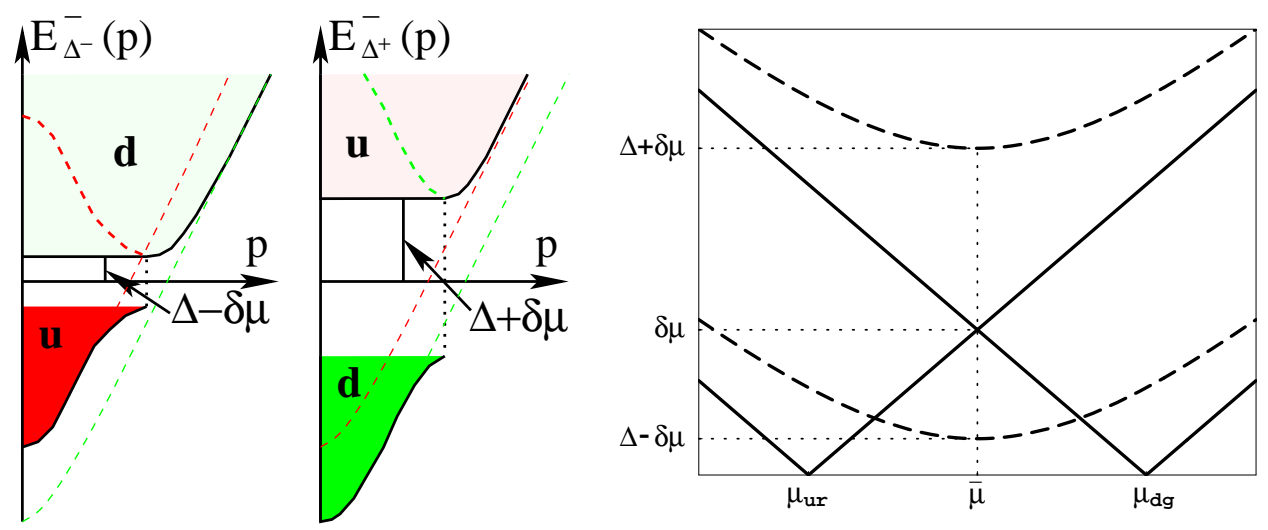

Fig. 3. The schematic modification of the red and green quark dispersion relations in the $2 \mathrm{SC}$ phase (left panel), and the resulting quasiparticle dispersion relations at low energies (right panel).

\subsection{Quasiparticle spectra in $2 S C$ and g2SC phase}

In the previous subsections, we established that the stable ground state in neutral quark matter is the g2SC phase, provided the coupling constant is neither too weak nor too strong $\left(G_{D}^{(1)} \leq G_{D} \leq G_{D}^{(2)}\right)$. However, these calculations alone may not be sufficient to understand the physical properties of such a phase. To compensate this limitation, we find it very useful to study the nature of the fermionic quasiparticles in the g2SC phase.

It is instructive to start with the case of the ordinary 2SC phase $(\Delta>\delta \mu)$. All four fermionic quasiparticles that result from the Cooper pairing of the red and green quarks are gaped (note that, with the conventional choice of the gap pointing in the anti-blue direction in color space, the spectra of the blue up and blue down quarks are not affected by the pairing dynamics).

The corresponding spectra are shown schematically in Fig. 3. The left panel gives a qualitative picture of the dispersion relations of the pairing quarks, see Eq. (10). The dark (light) shaded regions below (above) $E=0$ line represent the filled (empty) quark states. By combining the lines lying above $E=0$ with the reflections of the lines lying below $E=0$, one obtains the complete dispersion relations of the fermionic quasiparticles in the $2 \mathrm{SC}$ phase. The low energy slices of these relations are shown in the right panel by dashed lines. For comparison, the dispersion relations of quasiparticles in free theory are also shown in the same panel by solid lines.

It is interesting to observe how the nature of quasiparticles changes gradually when one goes from the region of small momenta to the region of large momenta. From Fig. 3, we see that the quasiparticles with the larger (smaller) gap are nearly identical with the down (up) quark holes at small momenta, $p \ll \bar{\mu}$, and resemble the up (down) quarks at large momenta, $p \gg \bar{\mu}$. Around 

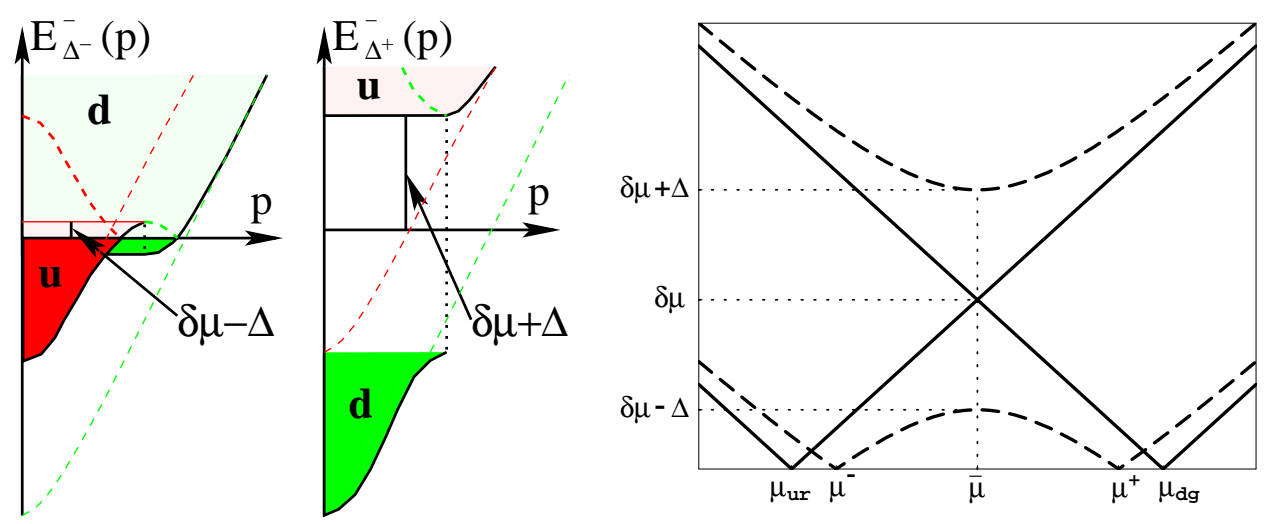

Fig. 4. The schematic modification of the red and green quark dispersion relations in the g2SC phase (left panel), and the resulting quasiparticle dispersion relations at low energies (right panel).

the middle point, $p \simeq \bar{\mu}$, the quark spectrum is strongly modified by the appearance of the gap. Thus, the quasiparticles represent presumably a mixture of different types of quarks and holes.

Now, let us turn to the quasiparticle spectra in the g2SC phase. Graphically, these are shown in Fig. 4 which has the same layout as Fig. 3. The left panel shows the qualitative dispersion relations of quarks in the case of $\Delta<\delta \mu$, see Eq. (10), while the right panel gives the low energy slice of the corresponding quasiparticle dispersion relations (dashed lines).

As one would expect, far outside the pairing region, $p \simeq \bar{\mu}$, the quasiparticle dispersion relations are similar to those in the 2SC phase. Also, around $p \simeq \bar{\mu}$, the gaped (double-degenerate) quasiparticle resembles the (doubledegenerate) quasiparticle with larger value of the gap in the $2 \mathrm{SC}$ phase. This is the quasiparticle that interpolates between the down quark holes at small momenta, $p \ll \bar{\mu}$, and the up quarks at large momenta, $p \gg \bar{\mu}$. The other (double-degenerate) quasiparticle is gapless. The energy of this latter vanishes at two values of momenta $p=\mu^{-}$and $p=\mu^{+}$where $\mu^{ \pm} \equiv \bar{\mu} \pm \sqrt{(\delta \mu)^{2}-\Delta^{2}}$.

The most remarkable property of the quasiparticle spectra in the g2SC phase, see the right panel in Fig. 4, is that the low energy excitations $(E \ll \delta \mu-\Delta)$ are very similar to those in the normal phase represented by solid lines. The only difference is that the values of the chemical potentials of the up and down quarks $\mu_{u r}=\mu_{u g}$ and $\mu_{d g}=\mu_{d r}$ are replaced by the values $\mu^{-}$and $\mu^{+}$, respectively. This observation suggests, in particular, that the low energy (large distance scale) properties of the g2SC phase should look similar to those in the normal phase. In the following subsection, we use this argument to justify that the Meissner effect might be absent in the g2SC phase. 


\subsection{Gluon properties in g2SC phase}

The main purpose for studying the NJL model in Eq. (1) is to understand the properties of dense quark matter under conditions that may exist inside compact stars. Indeed, this model should be adequate for getting an insight into the pairing dynamics of quarks and into some thermodynamic properties of quark matter. However, the NJL model lacks gluons. As reflection of this, it possesses the global instead of gauged color symmetry. The consequence is that there appear five Nambu-Goldstone (NG) bosons in a ground state of the model when the color symmetry is broken. In QCD, there is no room for such NG bosons.

Perhaps, the NJL model can be thought of as the low energy theory of QCD in which the gluons, as independent degrees of freedom, are integrated out. The gluons could be reintroduced back by gauging the color symmetry in the Lagrangian density in Eq. (1), providing a semirigorous framework for studying the effect of the Cooper pairing on the physical properties of gluons.

Gluon properties in the 2SC phase of dense quark matter without the mismatch of the Fermi momenta of the up and down quarks have been studied in detail [26,27]. A similar quantitative analysis could also be performed in the case of a nonzero mismatch parameter $\delta \mu$. Technically, this is not easy, and it is left for a future study. Here we argue, nevertheless, that some basic properties of gluons could be understood even without such an explicit calculation.

At large densities, gluons become quasiparticles with rather complicated dispersion relations. Typically, the only propagating (i.e., weakly damped) modes of gluons in dense medium are plasmons (see, however, Ref. [28] where other interesting modes of dense quark matter are discussed). As in the case of metals, the minimum value of the frequency of plasmons is called the plasma frequency. Since its value in dense quark matter is of order $\sqrt{\alpha_{s}} \mu$, the plasmons could be viewed as "high energy" modes that exist not only in the normal phase, but also in the 2SC and g2SC phases. The reason is that the properties of these high energy modes should not change much by the modification of the low energy quasiparticle spectra in broken phases.

It is clear that the gluon self-energy carries information not only about the propagating modes, but also about highly damped modes and various screening effects. The Meissner effect and the Debye screening are among them. It is important to mention that these types of large distance scale properties are determined to large extent by the low energy excitations. Therefore, they are very sensitive to the (dis-)appearance of gaps in quasiparticle spectra.

First, let us discuss the Debye screening which is the property of the gluons of the electric type (i.e., spatially longitudinal gluons). In the case of the 
2SC phase, this is studied in Ref. [26]. It is shown that the three gluons of the unbroken $S U(2)_{c}$, unlike the other five gluons of the original $S U(3)_{c}$, do not experience the Debye screening in the far infrared region. This is the consequence of the absence of gapless quasiparticles in the 2SC ground state that interact with the gluons of the unbroken $S U(2)_{c}$. Now, this property is no longer valid in the gapless phase studied here. One should expect, therefore, that all gluons of the original $S U(3)_{c}$ are subject to the Debye screening. Of course, the values of the Debye masses for different gluons need not be the same.

A similar line of arguments may also be given in relation to the Meissner effect that affects five magnetic gluons (i.e., spatially transverse gluons). In this case, it is the appearance of a nonzero gap that leads usually to Meissner masses for the gluons [26,27]. We remind that the three gluons of the unbroken $S U(2)_{c}$ do not experience the Meissner effect. It is reasonable to suggest that, even with a nonzero mismatch parameter, five magnetic gluons in the 2SC phase $(\Delta>\delta \mu)$ should still experience the Meissner effect. Indeed, all those quasiparticles that were gaped at $\delta \mu=0$ still remain gaped.

In the case of the g2SC phase $(\Delta<\delta \mu)$, there appear gapless quasiparticles in the low energy sector of the theory, see Fig. 4. If the value of the quantity $\delta \mu-\Delta$ is not vanishingly small, the low energy excitations $(E \lesssim \delta \mu-\Delta)$ in the g2SC phase look similar to the excitations in the normal phase in which the values of the chemical potentials are shifted slightly. Moreover, even the values of the density of states at the corresponding "effective" Fermi surfaces, i.e., at $p=\mu^{-}$and $p=\mu^{+}$, remain similar. By making use of the dispersion relations in Eq. (10), we derive

$$
\left.\frac{d N}{d E}\right|_{p=\mu^{-}}=\frac{2 \delta \mu\left(\mu^{-}\right)^{2}}{\sqrt{(\delta \mu)^{2}-\Delta^{2}}},\left.\quad \frac{d N}{d E}\right|_{p=\mu^{+}}=\frac{2 \delta \mu\left(\mu^{+}\right)^{2}}{\sqrt{(\delta \mu)^{2}-\Delta^{2}}}
$$

Taking this into account, we may conclude that the large distance properties of the magnetic gluons in the g2SC phase should be the same as in the normal phase. This suggests, in particular, that there is no color Meissner effect in such a phase. This would be very unusual because the diquark gap is nonzero, and the Anderson-Higgs mechanism is expected to occur. Sometimes, this latter is identified with the Meissner effect. We emphasize, however, that the two effects are not the same. Of course, the Anderson-Higgs mechanism is realized in the g2SC phase in the following sense. The five NG bosons, related to the breaking of the global color symmetry, are unphysical in the g2SC phase. In other words, they are "eaten" by the gluons. This conclusion can be reached by using the gauge freedom in QCD that allows to get rid of the NG bosons (the corresponding gauge is called unitary). 
Here, it is also instructive to mention that, in contrast to models with unbroken Lorentz symmetry, the gauge bosons in dense (or hot) medium have propagating (massive) longitudinal modes even in the normal phase. Thus, the intuitive picture, being popular in particle physics, that connects the Anderson-Higgs mechanism with the appearance of nonzero masses for gauge bosons is not directly generalized to dense (or hot) medium. In the case of the g2SC phase, it might not be adequate even when applied to the appearance of the Meissner mass for magnetic gluons.

\subsection{Spin-1 condensation}

The fact that the low energy fermionic quasiparticles in the g2SC phase look nearly the same as in the normal phase has yet another consequence. As we discuss below, there should appear two additional spin-1 condensates on top of the g2SC ground state. In its turn, this should lead to the opening of small gaps around the points $p=\mu^{-}$and $p=\mu^{+}$. Thus, strictly speaking, there will be no gapless excitations in the quasiparticle spectrum at zero temperature.

Our argument here is based on the following simple observation. Around the effective Fermi momenta $p=\mu^{-}$and $p=\mu^{+}$, the gapless quasiparticles are double degenerate. This is the reflection of the degeneracy with respect to the red and green colors. Then, by taking into account the high degeneracy of states at $p=\mu^{-}$and $p=\mu^{+}$and the attraction in the color antisymmetric channel, we conclude that the Cooper instability should develop in the g2SC ground state around both points $p=\mu^{-}$and $p=\mu^{+}$. This instability is removed spontaneously by the appearance of two "secondary" condensates of Cooper pairs. In view of the Pauli principle, these are spin- 1 condensates of the so-called polar phase. The spatial rotation symmetry is broken in the presence of such a condensation [8].

Since spin-1 condensates are much weaker than the spin-0 condensates, this additional condensations add a fine structure on the background of the g2SC phase. In practical terms, it means that many thermodynamic quantities, such as the energy density, the pressure and the particle densities should change very little. At the same time, other properties, such as the specific heat, the magnetic field penetration depth and various transport coefficients could be modified considerably by the appearance of the spin-1 condensates. For example, the presence of these condensates will result in the color as well as electromagnetic Meissner effect at sufficiently low temperatures [9].

Before concluding this subsection, we would like to mention that an additional spin-1 condensation of the blue up and down quarks is not expected in the model at hand. Even if there is an attraction in a flavor antisymmetric channel 
(e.g., provided by instantons), the Fermi momenta of the blue up and down quarks are badly mismatched. This is the general outcome of enforcing the $\beta$-equilibrium condition and the local neutrality in nonstrange quark matter.

\section{Gapless 2SC at finite temperature}

In this section, we study the properties of the g2SC phase at finite temperature. As we shall see, the interplay of the neutrality condition and the solution to the gap equation produces many unusual properties of quark matter that have no analogue in well known systems. One of our most intriguing observation will be that the ratio of the critical temperature to the value of the gap at zero temperature is not a universal number in the g2SC phase. In fact, depending on the coupling constant, this ratio could be arbitrarily large. The other striking property is related to a nonmonotonic dependence of the order parameter on temperature. In the most extreme case, for example, a nonzero gap can appear at finite temperature even it was exactly zero at $T=0$.

In a superconducting system, when one increases the temperature at a given chemical potential, thermal motion will eventually break up the quark Cooper pairs. In the weakly interacting Bardeen-Copper-Schrieffer (BCS) theory, the transition between the superconducting and normal phases is usually of second order. The ratio of the critical temperature $T_{c}^{\mathrm{BCS}}$ to the zero temperature value of the gap $\Delta_{0}^{\mathrm{BCS}}$ is a universal value [29]

$$
r_{\mathrm{BCS}}=\frac{T_{c}^{\mathrm{BCS}}}{\Delta_{0}^{\mathrm{BCS}}}=\frac{\mathrm{e}^{\gamma_{E}}}{\pi} \approx 0.567,
$$

where $\gamma_{E} \approx 0.577$ is the Euler constant. In the conventional 2SC phase of quark matter with equal densities of the up and down quarks, the ratio of the critical temperature to the zero temperature value of the gap is also the same as in the BCS theory [3]. In the spin-0 color flavor locked phase as well as in the spin-1 color spin locked phase, on the other hand, this ratio is larger than BCS ratio by the factors $2^{1 / 3}$ and $2^{2 / 3}$, respectively. These deviations are related directly to the presence of two different types of quasiparticles with nonequal gaps [30].

This commonly accepted picture of the finite temperature effects in superconducting phases changes drastically in the case of dense quark matter when the $\beta$-equilibrium and the local neutrality conditions are enforced. Below, we study this in detail. 


\subsection{Gap equation and charge neutrality condition}

Here we consider the gap equation and charge neutrality conditions in the g2SC phase at finite temperature. Because of additional technical complications appearing at finite temperature, most of the results that follow will be obtained by numerical computation. It is important to keep in mind, however, that our approach remains conceptually the same as that at zero temperature.

Before studying the gap equation (20), we recall that the right hand side of this equation is a function of the three chemical potentials $\mu, \mu_{e}$ and $\mu_{8}$. The values of $\mu_{e}$ and $\mu_{8}$ are not the free parameters in neutral quark matter. They are determined by satisfying the two conditions in Eq. (15). In our analysis, on the other hand, it is very convenient to enforce only the color charge neutrality by choosing the function $\mu_{8}\left(\mu, \mu_{e}, \Delta\right)$ properly. As for the electrical chemical potential $\mu_{e}$, it is treated as an independent parameter at intermediate stages of calculation. Only at the very end its value is adjusted to make the quark matter also electrically neutral.

As in the case of zero temperature, the resulting color chemical potential $\mu_{8}$ is much smaller than the other two chemical potentials in neutral quark matter. This, of course, is not surprizing. Small nonzero values of $\mu_{8}$ [typically, $\left.\mu_{8} \sim \Delta^{2} / \mu\right]$ are required only because an induced color charge of the diquark condensate should be compensated. Therefore, the smallness of $\mu_{8}$ is protected by the smallness of the order parameter. The numerical calculations show, in fact, that even the approximation $\mu_{8}=0$ does not modify considerably the exact solutions to the gap equation and to the electrical charge neutrality condition. In practice, we either tabulate the function $\mu_{8}\left(\mu, \mu_{e}, \Delta\right)$ for a given set of parameters, or determine it numerically in the vicinity of the ground state solution.

The solutions to the gap equation (20) for several different values of temperature $(T=0,20,40,50.2$ and $60 \mathrm{MeV})$ are shown graphically in Fig. 5 (solid lines). The values of the temperature are marked along the curves. The results are plotted in the $\left(\mu_{e}, \Delta\right)$-plane. In computation we kept the quark chemical potential fixed, $\mu=400 \mathrm{MeV}$, and used the diquark coupling constant $G_{D}=\eta G_{S}$ with $\eta=0.75$. This is the same choice that we used at zero temperature in Fig. 1.

Now let us briefly discuss the temperature dependence of the solution. It is not very surprizing that the shape of the graphical solution is smoothed with increasing the temperature. The same applies to the disappearance of the double-branch structure of the solution at a finite value of the temperature, $T_{s} \simeq 30 \mathrm{MeV}$. Indeed, in a model that treats the electrical chemical potential as a free parameter, this value of the temperature $T_{s}$ marks the expected 


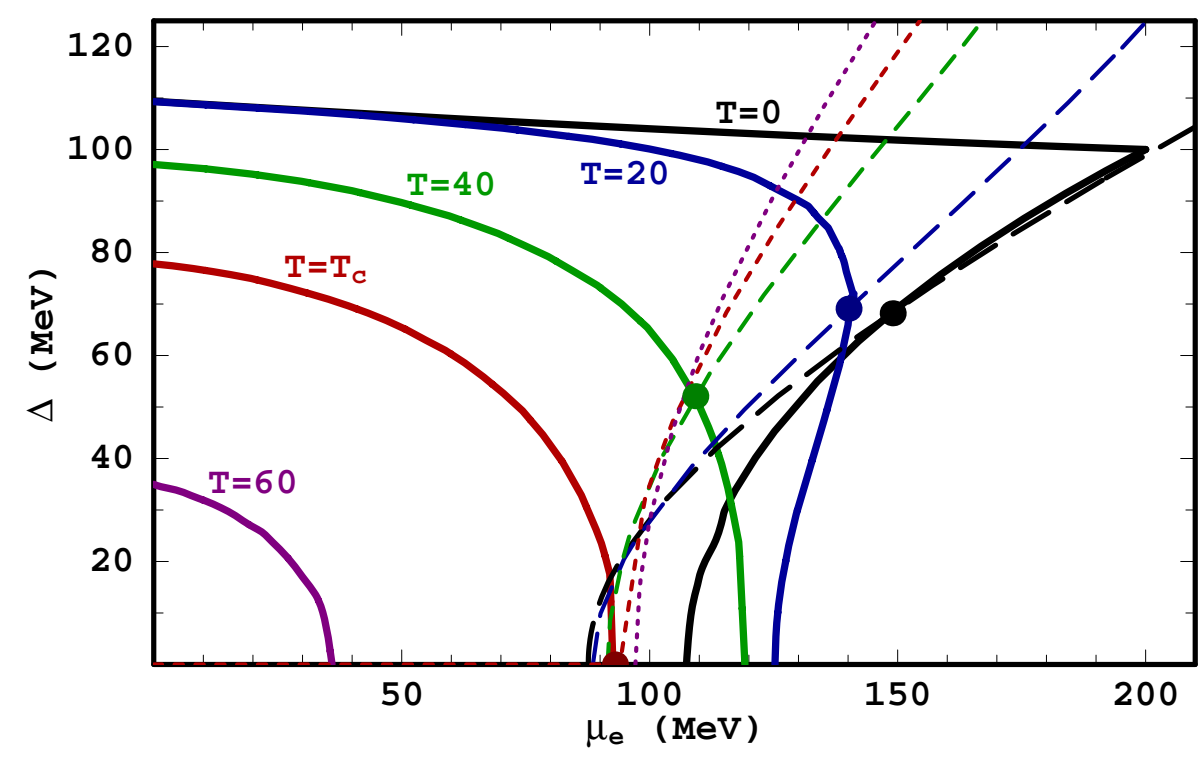

Fig. 5. The solutions to the gap equation (solid lines) and the neutrality condition (dashed lines) calculated for several values of temperature. The results are plotted for $\mu=400 \mathrm{MeV}$ and the diquark coupling constant $G_{D}=\eta G_{S}$ with $\eta=0.75$. In this case, $T_{c} \approx 50.2 \mathrm{MeV}$.

switch of two regimes. Namely, while the phase transition controlled by the $\mu_{e}$ parameter (i.e., neutrality is not required) is a first order phase transition at $T<T_{s}$, it becomes a second order phase transition at $T>T_{s}$.

We also observe that the value of the electrical chemical potential at the point where the solution to the gap equation intersects with the $\mu_{e}$-axis has a nonmonotonic dependence on the temperature. With increasing temperature, this value increases first and, after reaching some maximum value, starts to decrease and goes down to zero eventually. When the interplay with the neutrality condition is taken into account later, this simple property of the solution would produce rather unusual physical results.

We note that the temperature dependence of the solutions to the gap equation is very similar to the dependence found by Sarma in a solid state physics analogue of the g2SC phase [25]. Of course, there was no analogue of the neutrality condition in the system studied in Ref. [25]. Therefore, the mentioned similarity does not extend to the complete analysis of the g2SC phase that follows.

In Fig. 5, we also show the neutrality lines (dashed lines) for the same values of temperature $(T=0,20,40,50.2$ and $60 \mathrm{MeV})$. The convention is that the lengths of the dashes decreases with increasing the value of the temperature. As we see, with increasing temperature, the neutrality line gets steeper while its intersection point with the $\mu_{e}$-axis moves towards larger values of $\mu_{e}$. The points of intersection of these neutrality lines with the lines of solutions to the 


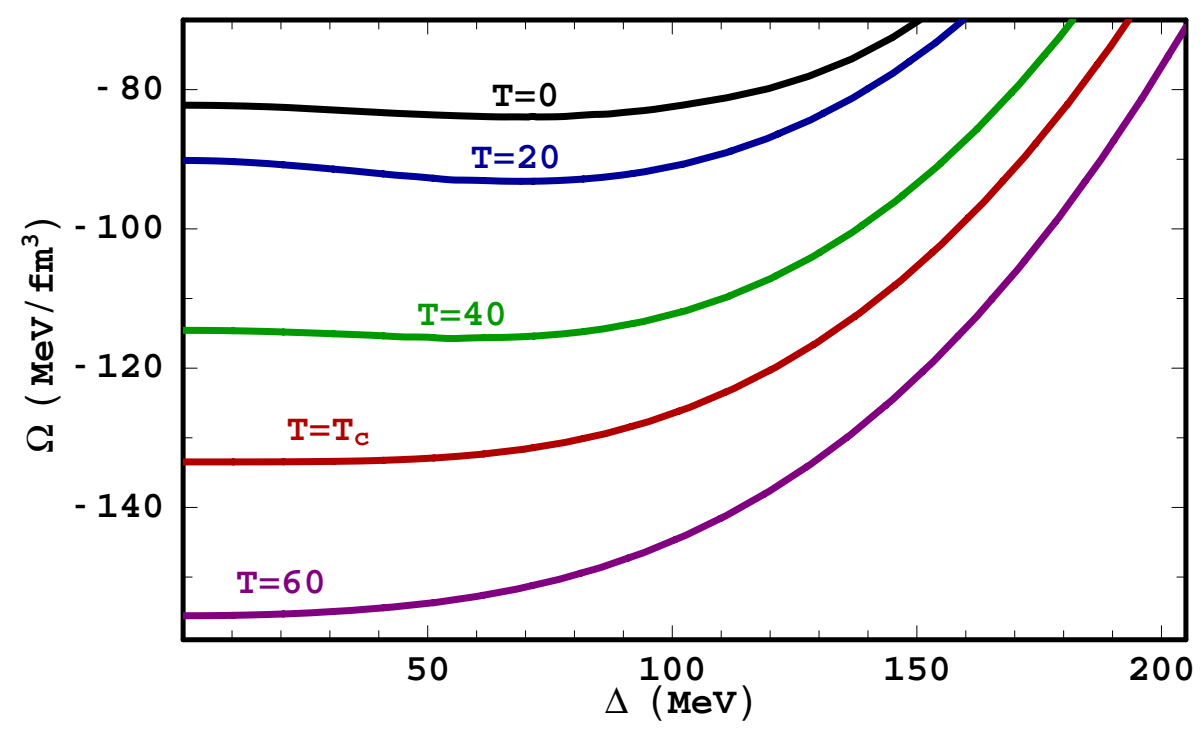

Fig. 6. The thermodynamic potential of neutral quark matter as a function of the diquark gap $\Delta$ calculated for several values of temperature.

gap equation, when they exist, are shown as well.

As in the case of zero temperature, see Subsec. 3.3 and Fig. 2, we need to show that the points of intersections of the gap solutions with the corresponding neutrality lines in Fig. 5 represent the ground state of the neutral quark matter. To this end, we calculate the dependence of the thermodynamic potential on the value of the gap $\Delta$. Since the charge neutrality condition is satisfied only along the neutrality line, we restrict the thermodynamic potential only to this line. The numerical results for several values of the temperature are shown in Fig. 6.

The results at $\mu=400 \mathrm{MeV}$ and $\eta=0.75$ show that, for any $T<T_{c} \approx 50.2$ $\mathrm{MeV}$, the thermodynamic potential has the global minimum away from the origin, meaning that the corresponding ground state develops a nonzero expectation value of $\Delta(T)$. Needless to say that this is the same value that one extracts from the location of the geometrical construction in Fig. 5. The expectation value $\Delta(T)$ disappears gradually when the temperature approaches $T_{c}$ from below. This is an indication of the second order phase transition. At temperature higher than $T_{c}$, the ground state of neutral quark matter is the normal phase with $\Delta(T)=0$.

\subsection{Temperature dependence of the gap}

The temperature dependence of the gap is obtained by numerical solution of the gap equation (20) together with the two charge neutrality conditions in Eq. (15). Of course, this is equivalent to the geometrical construction used in 


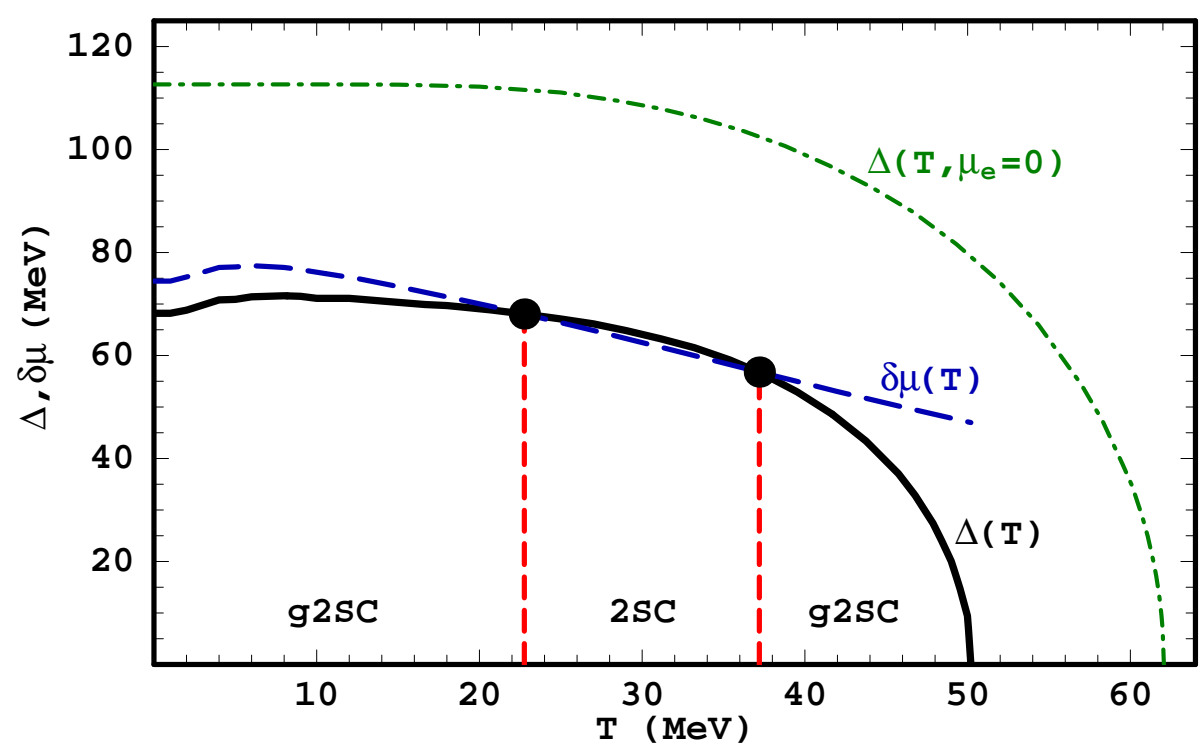

Fig. 7. The temperature dependence of the diquark gap (solid line) and the value of $\delta \mu \equiv \mu_{e} / 2$ (dashed line) in neutral quark matter. For comparison, the diquark gap in the model with $\mu_{e}=0$ and $\mu_{8}=0$ is also shown (dash-dotted line). The results are plotted for $\mu=400 \mathrm{MeV}$ and $\eta=0.75$.

Fig. 5, where the intersection points of two types of lines determine the values of the gaps in the ground state.

The typical results for the default choice of parameters $\mu=400 \mathrm{MeV}$ and $\eta=0.75$ are shown in Fig. 7. Both the values of the diquark gap (solid line) and the mismatch parameter $\delta \mu=\mu_{e} / 2$ (dashed line) are plotted. One very unusual property of the shown temperature dependence of the gap is the nonmonotonic behavior. Only at sufficiently high temperatures, the gap is a decreasing function. In the low temperature region, $T \lesssim 10 \mathrm{MeV}$, however, it increases with temperature. For comparison, in the same figure, the diquark gap in the model with $\mu_{e}=0$ and $\mu_{8}=0$ is also shown (dash-dotted line). This latter has the standard BCS shape.

Another interesting thing regarding the temperature dependences in Fig. 7 appears in the intermediate temperature region, $22.5 \lesssim T \lesssim 37 \mathrm{MeV}$. By comparing the values of $\Delta(T)$ and $\delta \mu$ in this region, we see that the g2SC phase is replaced by a "transitional" $2 \mathrm{SC}$ phase there. Indeed, the energy spectrum of the quasiparticles even at finite temperature is determined by the same relations in Eqs. (8) and (10) that we used at zero temperature. When $\Delta>\delta \mu$, the modes determined by Eq. (10) are gaped. Then, according to our standard classification, the ground state is the 2SC phase.

It is fair to say, of course, that the qualitative difference of the g2SC and $2 \mathrm{SC}$ phases is not so striking at finite temperature as it is at zero temperature. This difference is particularly negligible in the region of interest where temperatures 


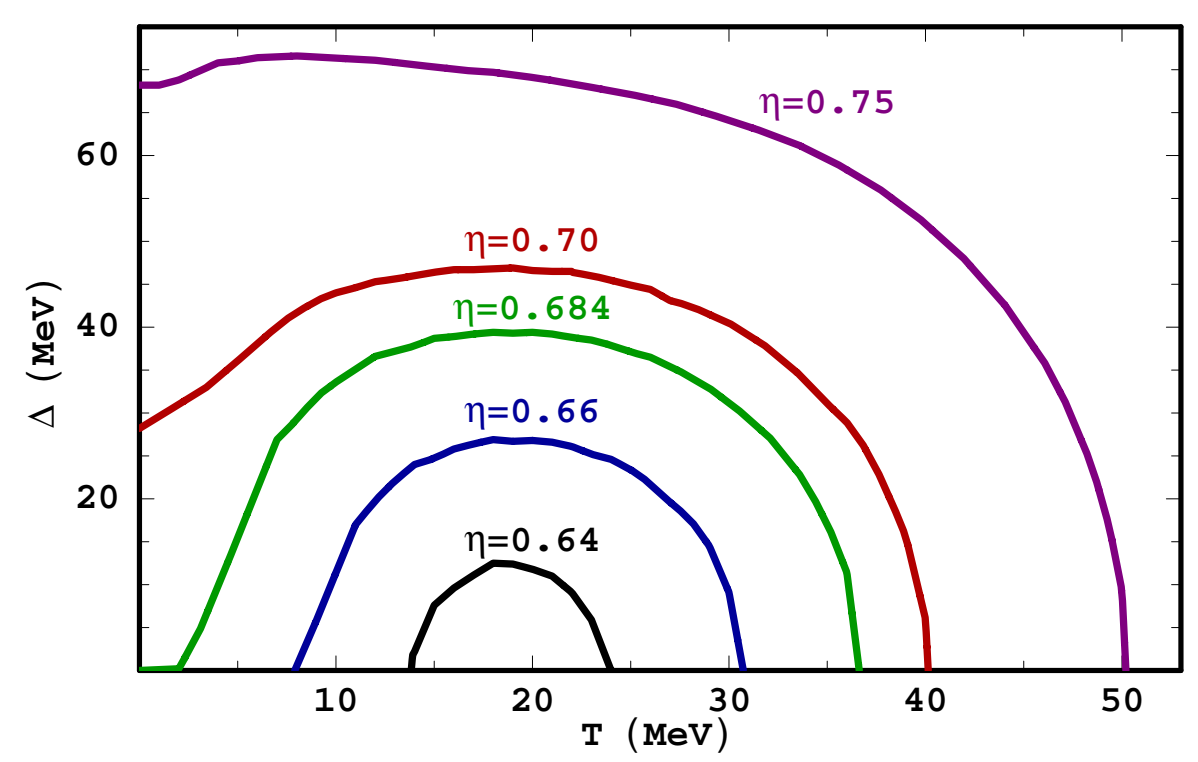

Fig. 8. The temperature dependence of the diquark gap in neutral quark matter calculated for several values of the diquark coupling strength $\eta=G_{D} / G_{S}$.

$22.5 \lesssim T \lesssim 37 \mathrm{MeV}$ are considerably larger than the actual value of the smaller gap, $\Delta-\delta \mu$. By increasing the value of the coupling constant slightly, however, the transitional 2SC phase can be made much stronger and the window of intermediate temperatures can become considerably wider. In either case, we find it rather unusual that the g2SC phase of neutral quark matter is replaced by a transitional 2SC phase at intermediate temperatures which, at higher temperatures, is replaced by the g2SC phase again.

It appears that the temperature dependence of the diquark gap is very sensitive to the choice of the diquark coupling strength $\eta=G_{D} / G_{S}$ in the model at hand. This is not surprising because the solution to the gap equation is very sensitive to this choice. The resulting interplay of the solution for $\Delta$ with the condition of charge neutrality, however, is very interesting. This is demonstrated by the plot of the temperature dependence of the diquark gap calculated for several values of the diquark coupling in Fig. 8.

The most amazing are the results for weak coupling. It appears that the gap function could have sizable values at finite temperature even if it is exactly zero at zero temperature. This possibility comes about only because of the strong influence of the neutrality condition on the ground state preference in quark matter. Because of the thermal effects, the positive electrical charge of the diquark condensate is easier to accommodate at finite temperature. This opens a possibility of the Cooper pairing that is forbidden at zero temperature.

We should mention that somewhat similar results for the temperature dependence of the gap were also obtained in Ref. [31] in a study of the asymmetric nuclear matter. The asymmetry parameter in nuclear matter $\alpha=$ 
$\left(\rho_{n}-\rho_{p}\right) /\left(\rho_{n}+\rho_{p}\right)$ (where $\rho_{n}$ and $\rho_{p}$ are the neutron and proton densities, respectively) plays a role similar to the coupling strength $\eta$ in the quark matter system.

\subsection{Nonuniversal ratio $T_{c} / \Delta_{0}$}

In the preceding subsection, we saw that the temperature dependence of the gap in the g2SC phase is very different from the standard benchmark result in the BCS theory. One of the main properties of the BCS temperature dependence is a universal value of the ratio of the critical temperature $T_{c}$ to the value of the gap at zero temperature $\Delta_{0}$, see Eq. (42). It is instructive, therefore, to calculate the same quantity in the g2SC phase of neutral quark matter.

Let us start from a simple exercise, and consider the results plotted in Fig. 7 more carefully. First of all, we find that $T_{c}^{2 S C} \approx 62.06 \mathrm{MeV}$ and $\Delta_{0}^{2 S C}=109.4$ $\mathrm{MeV}$ when there is no mismatch parameters in the model (i.e., $\mu_{e}=0$ and $\mu_{8}=0$ represented by dash-dotted line). It is not very surprizing that the ratio of interest $r_{2 S C} \approx 0.567$ is in agreement with the BCS result. Now, if we check the results for the gap function in the g2SC phase (solid line), we find that $T_{c}^{g 2 S C} \approx 50.2 \mathrm{MeV}$ and $\Delta_{0}^{g 2 S C} \approx 68.2 \mathrm{MeV}$. Thus, the ratio is $r_{g 2 S C} \approx 0.7357$ which is considerably larger than the $2 \mathrm{SC}$ result.

It appears that the real situation is even more interesting. The result for the ratio $r_{g 2 S C} \approx 0.7357$ is not universal. In fact, its value depends very much on the diquark coupling constant and, moreover, it can even be arbitrarily large. This last statement may not be so unexpected if we recall the temperature dependences of the gap shown in Fig. 8.

The numerical results for the ratio of the critical temperature to the zero temperature gap in the g2SC case as a function of the diquark coupling strength $\eta=G_{D} / G_{S}$ are plotted in Fig. 9. The dependence is shown for the most interesting range of values of $\eta=G_{D} / G_{S}, 0.68 \lesssim \eta \lesssim 0.81$, which allows the g2SC stable ground state at zero temperature, see the discussion in Subsec. 3.4. When the coupling gets weaker in this range, the zero temperature gap vanishes gradually. As we saw from Fig. 8, however, this does not mean that the critical temperature vanishes too. Therefore, the ratio of a finite value of $T_{c}$ to the vanishing value of the gap can become arbitrarily large. In fact, it remains strictly infinite for a range of couplings. 


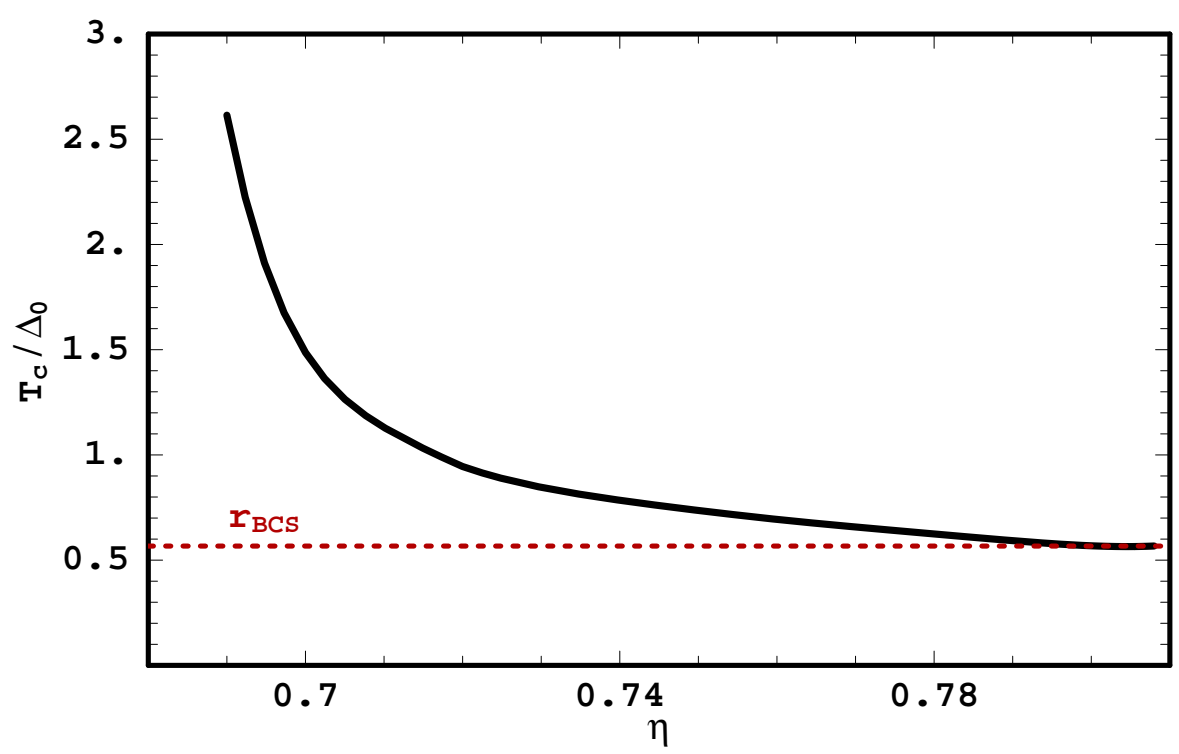

Fig. 9. The ratio of the critical temperature to the zero temperature gap in neutral quark matter as a function of the coupling strength $\eta=G_{D} / G_{S}$.

\section{Conclusion}

In this paper, we presented a detailed study of the zero and finite temperature properties of the gapless color superconducting phase of neutral two-flavor quark matter in $\beta$-equilibrium. The spectrum of quasiparticles in this phase has four gapless and only two gaped fermionic modes, see Eqs. (8)-(10) with $\Delta<\mu_{e} / 2$. This is in contrast to the conventional 2SC phase that has only two gapless and four gaped quasiparticles, see the case with $\Delta>\mu_{e} / 2$. As we show, the choice of the ground state is predetermined by the strength of the diquark coupling. In strong coupling regime, the 2SC survives even under the constrain of the charge neutrality and $\beta$-equilibrium. When the coupling is not very strong, the 2SC ground state is replaced by the g2SC phase. Finally, at weak coupling, the stable ground state corresponds to the normal phase.

In the g2SC phase, like in the conventional 2SC phase, the original color symmetry $\mathrm{SU}(3)_{c}$ is broken down to $\mathrm{SU}(2)_{c}$. Despite that, the low energy spectrum of the gapless phase looks very similar to the spectrum in the normal quark matter. By the "normal quark matter" here we understand the mixture of the following four normal components: (i) red and green up quarks with an effective Fermi momentum $p_{F}^{u, \text { eff }}=\mu^{-}$, (ii) red and green down quarks with an effective Fermi momentum $p_{F}^{d, \text { eff }}=\mu^{+}$, (iii) blue up quarks with $p_{F}^{u b}=\mu_{u b}$ and (iv) blue down quarks with $p_{F}^{d b}=\mu_{d b}$. Needless to say that the similarity extends only in a small region of low energies, $E \lesssim \delta \mu-\Delta$. This similarity suggests that there is no color Meissner effect in this phase of matter, although the gauge symmetry is broken through the Anderson-Higgs mechanism. 
The same arguments regarding the resemblance of the low energy spectrum in the g2SC and normal phases of quark matter lead us to the conclusion that two "secondary" spin-1 condensates should develop on top of the gapless ground state. Indeed, the existence of highly degenerate gapless modes around the effective two Fermi surfaces should lead to the appearance of additional condensates, provided an attractive channel exists. Because of the red-green color degeneracy of the quasiparticles around $p_{F}^{u, \text { eff }}=\mu^{-}$and $p_{F}^{d, \text { eff }}=\mu^{+}$, the attractive antisymmetric channel should exist. As a result, two spin-1 gaps would open around the points where the gapless modes used to be.

Most likely, the appearance of the spin-1 gaps is a small effect on top of the g2SC ground state. Therefore, many thermodynamical properties of quark matter would not be affected by their presence. The transport properties, on the other hand, should be modified. In particular, five gluons of the original $\mathrm{SU}(3)_{c}$ gauge group should experience the color Meissner effect. In addition there will also be the electromagnetic Meissner effect [9].

At finite temperature, the properties of the g2SC phase are very unusual. One of the most striking results is that the ratio of the critical temperature to the gap at zero temperature is not a universal number. It depends on the choice of the coupling constant in the diquark channel. Moreover, the value of the ratio can become arbitrarily large with proper choice of the coupling. For a range of values, it is infinite. This is a simple consequence of the nonmonotonic temperature dependence of the diquark gap function on temperature. In an extreme case, the finite temperature gap can be nonzero even if the zero temperature gap is zero. To the best of our knowledge, the only other example of such a temperature behavior of an order parameter was obtained in Ref. [31] in a study of the asymmetric nuclear matter.

In nature, gapless 2SC quark matter could exist in compact stars. Indeed, this phase is neutral with respect to electric and color charges and satisfies the $\beta$-equilibrium condition by construction. If this is indeed the case, one may have a chance to detect the indirect signatures of its presence by deciphering the observational data from stars. The study of the physical properties of the g2SC phase in this paper is the first step in this direction. In future, one should also study the transport properties of the g2SC phase. For example, the knowledge of the neutrino emissivities and mean free paths would be crucial for the understanding of the cooling rates of stars with such a gapless phase in their interior.

As we mentioned earlier, some stable gapless phases may also be realized in the asymmetric nuclear matter [31] and in two-component mixtures of cold fermionic atoms [15]. The results of this paper might also be useful for a deeper understanding of the properties of such nonrelativistic systems. For example, the similarity of the spectrum of the low energy quasiparticles in the gapless 
phase and the normal matter seems to suggest that there is no superfluidity in the corresponding phases of cold atom mixtures. Of course, one should be careful before making a conclusive statement because the corresponding nonrelativistic systems are quite different from neutral quark matter.

Acknowledgements. The authors thank Prof. D.H. Rischke for stimulating discussions as well as H. Caldas and P. Bedaque for comments. I.A.S. would like to thank T. Schäfer for discussions. M.H. acknowledges the financial support from the Alexander von Humboldt-Foundation, and the NSFC under Grant Nos. 10105005, 10135030. The work of I.A.S. was supported by Gesellschaft für Schwerionenforschung (GSI) and by Bundesministerium für Bildung und Forschung (BMBF).

\section{References}

[1] B. C. Barrois, Nucl. Phys. B129, 390 (1977); S. C. Frautschi, in "Hadronic matter at extreme energy density", edited by N. Cabibbo and L. Sertorio (Plenum Press, 1980); D. Bailin and A. Love, Phys. Rep. 107, 325 (1984).

[2] D. T. Son, Phys. Rev. D 59, 094019 (1999); T. Schäfer and F. Wilczek, Phys. Rev. D 60, 114033 (1999); D. K. Hong, V. A. Miransky, I. A. Shovkovy, and L. C. R. Wijewardhana, Phys. Rev. D 61, 056001 (2000); S. D. H. Hsu and M. Schwetz, Nucl. Phys. B572, 211 (2000); W. E. Brown, J. T. Liu, and H.C. Ren, Phys. Rev. D 61, 114012 (2000).

[3] R.D. Pisarski and D.H. Rischke, Phys. Rev. D 61, 051501 (2000).

[4] I. A. Shovkovy and L. C. R. Wijewardhana, Phys. Lett. B 470, 189 (1999); T. Schäfer, Nucl. Phys. B575, 269 (2000).

[5] M. Alford, K. Rajagopal, and F. Wilczek, Phys. Lett. B 422, 247 (1998); R. Rapp, T. Schafer, E. V. Shuryak and M. Velkovsky, Phys. Rev. Lett. 81, 53 (1998).

[6] M. G. Alford, K. Rajagopal and F. Wilczek, Nucl. Phys. B537, 443 (1999).

[7] M. G. Alford, J. A. Bowers and K. Rajagopal, Phys. Rev. D 63, 074016 (2001); J. A. Bowers, J. Kundu, K. Rajagopal and E. Shuster, Phys. Rev. D 64, 014024 (2001); R. Casalbuoni, R. Gatto, M. Mannarelli and G. Nardulli, Phys. Rev. D 66, 014006 (2002); I. Giannakis, J. T. Liu and H. C. Ren, Phys. Rev. D 66, 031501 (2002); J. A. Bowers and K. Rajagopal, Phys. Rev. D 66, 065002 (2002).

[8] T. Schafer, Phys. Rev. D 62, 094007 (2000); M. G. Alford, J. A. Bowers, J. M. Cheyne and G. A. Cowan, Phys. Rev. D 67, 054018 (2003); M. Buballa, J. Hosek and M. Oertel, Phys. Rev. Lett. 90, 182002 (2003).

[9] A. Schmitt, Q. Wang and D. H. Rischke, nucl-th/0301090. 
[10] M. Alford and K. Rajagopal, JHEP 0206, 031 (2002).

[11] A. W. Steiner, S. Reddy and M. Prakash, Phys. Rev. D 66, 094007 (2002).

[12] I. Shovkovy and M. Huang, Phys. Lett. B 564, 205 (2003).

[13] E. Gubankova, W. V. Liu and F. Wilczek, Phys. Rev. Lett. 91, 032001 (2003).

[14] M. G. Alford, J. Berges and K. Rajagopal, Phys. Rev. Lett. 84, 598 (2000).

[15] W. V. Liu and F. Wilczek, Phys. Rev. Lett. 90, 047002 (2003).

[16] F. Neumann, M. Buballa, and M. Oertel, Nucl. Phys. A 714, 481 (2003).

[17] I. Shovkovy, M. Hanauske and M. Huang, Phys. Rev. D 67, 103004 (20003).

[18] P. F. Bedaque, H. Caldas and G. Rupak, cond-mat/0306694.

[19] K. Rajagopal and F. Wilczek, Phys. Rev. Lett. 86, 3492 (2001).

[20] M. Huang, P. F. Zhuang and W. Q. Chao, Phys. Rev. D 67, 065015 (2003).

[21] T. M. Schwarz, S. P. Klevansky and G. Papp, Phys. Rev. C 60, 055205 (1999).

[22] A. Mishra and H. Mishra, hep-ph/0306105.

[23] P. F. Bedaque, Nucl. Phys. A 697, 569 (2002); O. Kiriyama, S. Yasui and H. Toki, Int. J. Mod. Phys. E 10, 501 (2001).

[24] A. M. Clogston, Phys. Rev. Lett. 9, 266 (1962); B. S. Chandrasekhar, Appl. Phys. Lett. 1, 7 (1962).

[25] G. Sarma, J. Phys. Chem. Solids 24, 1029 (1963).

[26] D. H. Rischke, Phys. Rev. D 64, 094003 (2001).

[27] D. H. Rischke and I. A. Shovkovy, Phys. Rev. D 66, 054019 (2002).

[28] V. P. Gusynin and I. A. Shovkovy, Nucl. Phys. A 700, 577 (2002).

[29] J. R. Schrieffer, Theory of Superconductivity (Benjamin, New York, 1964).

[30] A. Schmitt, Q. Wang and D. H. Rischke, Phys. Rev. D 66, 114010 (2002);

D. H. Rischke, nucl-th/0305030

[31] A. Sedrakian and U. Lombardo, Phys. Rev. Lett. 84, 602 (2000). 